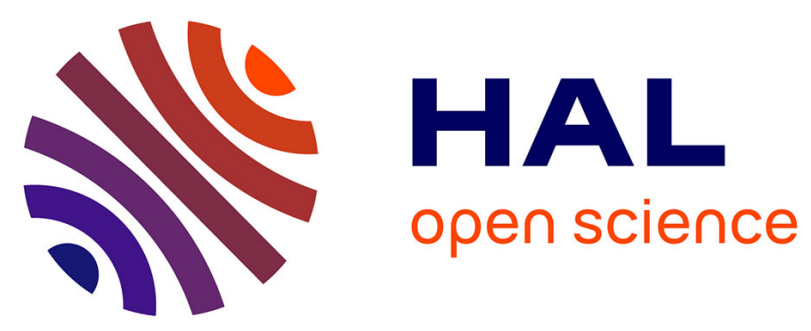

\title{
A quantitative cellular automaton model of multicellular spheroid tumour growth
}

\author{
Monika Joanna Piotrowska, Simon D. Angus
}

\section{To cite this version:}

Monika Joanna Piotrowska, Simon D. Angus. A quantitative cellular automaton model of multicellular spheroid tumour growth. Journal of Theoretical Biology, 2009, 258 (2), pp.165. 10.1016/j.jtbi.2009.02.008 . hal-00554577

\section{HAL Id: hal-00554577 \\ https://hal.science/hal-00554577}

Submitted on 11 Jan 2011

HAL is a multi-disciplinary open access archive for the deposit and dissemination of scientific research documents, whether they are published or not. The documents may come from teaching and research institutions in France or abroad, or from public or private research centers.
L'archive ouverte pluridisciplinaire HAL, est destinée au dépôt et à la diffusion de documents scientifiques de niveau recherche, publiés ou non, émanant des établissements d'enseignement et de recherche français ou étrangers, des laboratoires publics ou privés. 


\section{Author's Accepted Manuscript}

A quantitative cellular automaton model of in vitro multicellular spheroid tumour growth

Monika Joanna Piotrowska, Simon D. Angus

PII: $\quad$ S0022-5193(09)00067-8

DOI: $\quad$ doi:10.1016/j.jtbi.2009.02.008

Reference: $\quad$ YJTBI5461

To appear in: $\quad$ Journal of Theoretical Biology

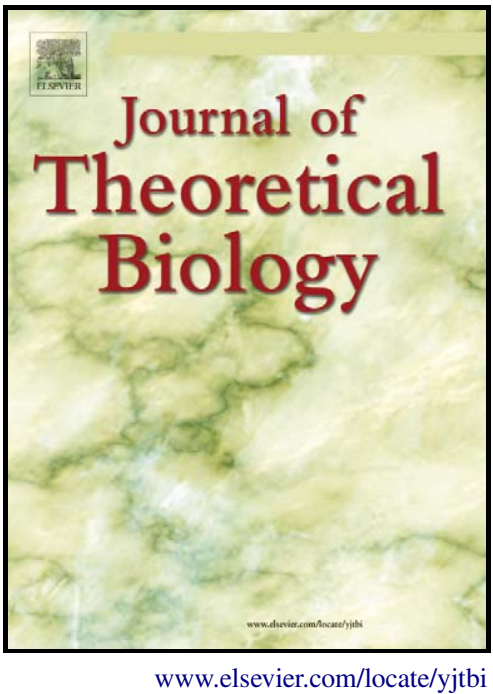

Received date: $\quad 13$ March 2008

Revised date: $\quad 15$ January 2009

Accepted date: $\quad 7$ February 2009

Cite this article as: Monika Joanna Piotrowska and Simon D. Angus, A quantitative cellular automaton model of in vitro multicellular spheroid tumour growth, Journal of Theoretical Biology (2009), doi:10.1016/j.jtbi.2009.02.008

This is a PDF file of an unedited manuscript that has been accepted for publication. As a service to our customers we are providing this early version of the manuscript. The manuscript will undergo copyediting, typesetting, and review of the resulting galley proof before it is published in its final citable form. Please note that during the production process errors may be discovered which could affect the content, and all legal disclaimers that apply to the journal pertain. 


\title{
A Quantitative Cellular Automaton Model of in vitro Multicellular Spheroid Tumour Growth
}

\author{
Monika Joanna Piotrowska* \& Simon D. Angus ${ }^{\dagger}$
}

(Dated: February 12, 2009)

\begin{abstract}
We report numerical results from a two dimensional Cellular Automaton (CA) model describing the dynamics of the in vitro cultivated multicellular spheroid obtained from EMT6/Ro (mammary carcinoma) cell line. Significantly, the CA model relaxes the often assumed one-to-one correspondence between cells and CA sites so as to correctly model the peripheral mitotic boundary region, and to enable the study of necrosis in large avascular tumours. By full calibration and scaling to available experimental data, the model produces with good accuracy experimentally comparable data on a range of bulk tumour kinetics and necrosis measures. Our main finding is that the metabolic production of $\mathrm{H}^{+}$ions is not sufficient to cause central necrosis prior to the sub-viable nutrient-deficient stage of tumour development being reached. Thus, the model suggests that an additional process is required to explain the experimentally observable onset of necrosis prior to the non-viable nutrient-deficient point being reached.
\end{abstract}

KEYWORDS: EMT6/Ro MAMMARY CARCINOMA; COMPUTATIONAL; NECROSIS.

${ }^{*}$ University of Warsaw, Department of Mathematics Informatics and Mechanics, Institute of Applied Mathematics and Mechanics, Banacha 2, 02 - 097 Warsaw, Poland. monika@mimuw.edu.pl

$\dagger$ Department of Economics, Monash University, Clayton 3800, VIC Australia. simon. angus@buseco.monash.edu.au. 


\section{INTRODUCTION}

Medical statistics continue to reveal the fact that benign and malignant tumours are the main diseases of the XXI century with over 1.7 million deaths attributed to cancer in Europe for 2006 alone (Ferlay et al., 2007). These data provide motivation for mathematicians, working together with physicists, to propose an increasingly wide spectrum of mathematical models to study different kinds of malignant tumours (Bellomo and Forni, 1994; Gatenby et al., 2005; Preziosi, 2003). Of these contributions, some have focused on describing and analysing the dynamics of bloodborne tumours (Andersen and Mackey, 2001; Moore and Lib, 2004) or soft-tissue tumours (Bodnar and Forys, 2005; Byrne and Chaplain, 1995, 1996b; Chaplain, 1996; Chaplain et al., 2001; Hatzikirou et al., 2005; Piotrowska, 2008; Piotrowska and Foryś, 2004), however in both cases, the aim of mathematical models and simulations is the same - to offer a better understanding of cancer dynamics which can be used to improve therapeutic outcomes.

It is well known that the key to restoring the cancer patient to health is the early detection of neoplastic changes in healthy tissue. Hence, in this paper we consider this early stage of tumour growth, namely, we study the growth and development of avascular, multicellular spheroids (MCS), i.e. solid symmetric tumours in the first stages of their development. Furthermore, since it has long been recognised that regions of cells in avascular tumours are susceptible to necrosis (unprogrammed cell death) (see Fig. 1) there has been much interest amongst experimentalists (Freyer $\underset{\text { about }}{\Leftarrow \text { Fig } 1}$

and Sutherland, 1985, 1986; Walenta et al., 2000) and theorists (Groebe and Mueller-

Klieser, 1996; Venkatasubramanian et al., 2006) alike in trying to understand the 
basis of this phenomenon, with an eye to utilising this process for therapeutic means. Hence, we have utilised several novel modeling approaches to faithfully re-produce experimental data on necrosis for one well-studied cell line and have provided further evidence for the basis, and progression, of necrosis. Specifically, we consider the in vitro cultivated EMT6/Ro mouse mammary tumour cell line. This line was selected on the one hand, because mammary tumours are named amongst the top three mortality causing cancers (Ferlay et al., 2007), and on the other, since a large amount of in vitro data is available in the literature for this particular cell line.

Our approach follows the encouraging results from what may be termed the computational oncology literature (e.g. Dormann and Deutsch (2002); Gerlee and Anderson (2007); Kansal et al. (2000a,b); Patel et al. (2001)) by implementing a 2D Cellular Automata (CA) model of avascular tumour growth. We differ substantially from previous approaches, however, in that we allow more than one cell to occupy each CA site as is consistent with lattice-gas CA implementations. We believe that this methodology represents the correct treatment of the CA setup since without it, one is constrained to treating the proliferating rim as a one cell width layer around the tumour mass. Furthermore, the 'many-to-one' assumption allows the implementation of a fully calibrated in silico tumour mass that can successfully grow to cell-count sizes on the order of $1 \times 10^{6}$ but with several orders of magnitude less numerical objects to handle. In this way, the implementable model produces realistic, experimentally comparable data on the onset and progression of necrosis. A theoretical interpretation of this assumption is also provided to encourage further investigation by the field. 
After full calibration and scaling of all experimentally available input data on cellular metabolism, diffusion, mitosis and cell death, we find that necrosis appears in our model due to sub-viable nutrient concentrations in the centre of the tumour and not, as has been suggested by some authors (e.g. Freyer and Sutherland (1986)), due to rising toxicity in the tumour mass. Indeed, we show that by including the diffusion of waste materials out of the tumour mass, central waste $\left(\mathrm{H}^{+}\right.$in our model $)$ concentrations are only sufficient to switch cells to quiescence rather than unprogrammed cell death (Casciari et al., 1992). Further results on the progression of the necrotic volume are included and compared to experimental results. Given that recent experimental data on nutrient concentrations prior to the onset of necrosis contradict these results (Walenta et al., 2000), the paper thus concludes that additional mechanisms for necrosis are required to be identified by the literature.

The paper is organized as follows: in Section 2 we outline the description of MCS growth model, Section 3 contains the parameter estimation, while in Section 4 the result of our computational simulations were compared with experimental data for multicellular spheroids of the EMT6/Ro tumour line cultivated in vitro. Finally, Section 5 contains the comparison of our model with other (discrete and continuous) models known from the literature. In this section the current limitations and potential applications of the present model for future research are discussed, as well. 


\section{Model Definition}

\subsection{The 2D Cellular Automaton}

We consider a 2D cellular automaton model comparable to those of, for instance, Patel et al. (2001) and Gerlee and Anderson (2007). Since it has been established that cells cultivated in the in vitro three-dimension (3D) like fashion behave differently to those that are kept as monolayers (Weaver et al., 1997), we approach the $2 \mathrm{D}$ automaton as a representative planar slice through a $3 \mathrm{D}$ spheroid-like tumour mass. For instance, nutrients are supplied to the growing tumour seed from CA sites beyond the boundary of occupied sites, mimicking the spheroid approach in the laboratory (as opposed to the planar substrate support approach as utilised in monolayer experiments). Subsequently, we use experimental data taken from in vitro spheroid studies as inputs to, and comparison with, our model, adjusting for the planar approach as necessary (e.g. calculation of saturated volume).

However, we differ significantly with all known previous CA tumour approaches by relaxing the perceived 'enforced' constraint of a 'one-to-one correspondence between automaton elements and physical cells' (Patel et al., 2001, p.319). Instead, in our approach, each automaton site is filled with a chosen packet of homogeneous cells (count $N$ ) (see Fig. 2), such that subsequent automaton updates occur at the $\underset{\substack{\text { about } \\ \text { Fig } 2}}{\text { a }}$

level of the site, rather than at the cellular level. Indeed, this approach is of wide inhere terested in statistical mechanics where it is often called coarse-graining (M. Kardar, 2007).

The reasons for this approach are two-fold. First, although it has been argued 
that the CA approach is perhaps the pre-eminent simulation approach for manyobject biological systems such as tumour growth due to its significantly smaller computational time compared to (say) apparently more realistic continuous interaction models (Drasdo, 2005), the fact remains that under a one-to-one assumption between cells and automaton sites, to model a biological system such as (even) pre-angiogenic tumours, one needs to implement a complex system with up to $10^{6}$ objects (cells). At this scale, even the CA approach is prohibitively time-consuming to carry out meaningful in silico experiments. Hence, a reduction in system objects is desirable purely due to implementation considerations.

However, the second reason for our approach is very important, and to our knowledge, has been overlooked in the biological simulation literature. Namely, by using the CA approach, the modeller must unavoidably assume a free-near-neighbour division restriction. That is, for an occupied parent site to successfully divide, a free neighbouring site (one of the orthogonal four, or Moore neighbourhood eight sites, depending on assumptions) must be available to place the daughter cell into. The reason for this is that in the CA setup, one assumes a binary 'occupied' or 'unoccupied' discretised world, such that occupancy by more than one object (cell) at any given site is illegal.

Whilst this 'legality' aspect of the CA approach has been acknowledged previously and has promoted a range of different approaches to cell mobility, what has not been acknowledged are the boundary conditions that this assumption entails. If one considers the boundary of a non-necrotic growing CA tumour (i.e. any boundary between occupied and unoccupied sites), the restriction implies that only sites at the 
interface can divide. An occupied site (i.e. a cell) just one site in from the tumour interface cannot divide because of the CA legality restriction just mentioned. Put in other words, the proliferative rim of such a one-to-one cell-to-site CA approach will be one cell wide, i.e. approximately $10 \mu \mathrm{m}$, by construction. It is known from the experimental literature that the proliferative rim (i.e. cells capable of dividing) is more likely at least on the order of $100 \mu \mathrm{m}$ (see Freyer and Sutherland (1985); Walenta et al. (2000)) and possibly even thicker than this. It is our contention that such a consideration is non-trivial and therefore that CA models must make allowances for such considerations if the approaches of computational oncology are to approach experimental accuracy.

Of course, the cost in applying (as we do) the 'many-to-one' assumption of cells per site, is that we increase the aggregation-level of the results of the model. In our approach, since we only study the tumour at the level of a site (rather than a cell), we must acknowledge that all cells in a given site are being effectively treated as an homogeneous population. However, as will be shown below, the implications of this assumption do not appear to adversely affect the outcomes of the model, and indeed, allow the very realistic computational study of larger tumours.

\subsection{CA Scaling: the many-to-one assumption}

To implement the many-to-one assumption as introduced above, each lattice site is taken to be of unit-cube dimension with side-length $u$ and containing $N$ cells (Fig. 2). The value of $u$ can be calculated from $N$ by utilising the cell packing density $(\rho)$ which has been reported to be equal to $3-5 \times 10^{8}$ cell per $\mathrm{cm}^{3}$ through 
the growth period (Freyer and Sutherland, 1985). Based on this result and taking $\rho=4 \times 10^{-4}$ cell. $\mu \mathrm{m}^{-3}$, the volume occupied by $N$ cells $V_{\text {cells }}=N \rho^{-1} \mu \mathrm{m}^{3}$ is first calculated, and then by assuming that a single lattice site is occupied by a filled cube of cells with volume $V_{\text {site }}=u^{3}$, one obtains an estimate for the site side-length $u=\left(V_{\text {site }}\right)^{\frac{1}{3}}=2\left(\frac{N}{\rho}\right)^{\frac{1}{3}}$ in $\mu \mathrm{m}$. In this way, the key determinant of lattice geometry and subsequent model scaling is the decision of $N$. As explained above, we have chosen a value for $N=400$ which results in a corresponding value for $u$ of $100 \mu \mathrm{m}$.

Indeed, a more detailed analysis of CA scaling can be made theoretically. If one assumes (as it is argued above when using the CA approach) that the mitotic region of the tumour is concentrated at the periphery of the tumour mass then we may consider the following master equation for the rate of tumour cell count $(C)$ with time

$$
\frac{d C}{d t}=k N \epsilon
$$

where $k$ is the rate constant (units $t^{-1}$ ), $N$ is the constant number of cells per CA site as defined above and $\epsilon$ is the number of $C A$ sites at the proliferation boundary of the tumour mass. Now for a 2D CA model, the proliferation boundary constitutes a circular loop of width and depth $u$, with radius $r$, and so, $\epsilon=2 \pi r / u$. Given that $C=N\left(\pi r^{2} / u^{2}\right)$ an expression for $r$ in terms of $C$ can be obtained, which leads to the differential equation

$$
\frac{d C}{d t}=2 k(\pi N C)^{\frac{1}{2}}
$$

which yields the expression (with the constant portion captured in $C_{0}$ ),

$$
C(t)=\pi N(k t)^{2}+C_{0}
$$


Similarly the evolution of the diameter with time is obtained immediately to be,

$$
d(t)=2 k(N / \rho)^{\frac{1}{3}} t+d_{0} .
$$

We see that the well known linear relationship between diameter and time is recovered in this model, and that a quadratic relationship appears in the total cell count with time evolution. Furthermore, it can be seen that the scaling assumption appears as a constant which can be used to re-scale any data into single cell per site, or many cell per site scenarios. Of course, this procedure is limited by the region over which the peripheral mitotic dynamics are expected. Thus, at very early time periods (where the CA model may not be well approximated by a circle/sphere) or in later periods (e.g. when the necrotic volume fraction is much larger than zero) this model may not apply. Similarly, the above analysis assumes that cells are stationary during one time step (or at least that the mean free path radius is zero) and so whilst appropriate for the present CA setup, it remains for the analysis of real spheroid growth dynamics to shed light on the validity of this assumption. Nevertheless, one should expect that CA models of tumour growth will yield these relationships and thus provide a basis for comparison of results of different scale. Furthermore, this simple theory provides a test of whether growth is constrained to the periphery in the mitotic dynamics of real spheroids (similar calculations can be made for the 3D environment). 


\subsection{Cellular Metabolism}

The model implements a more mature cellular metabolism module than has been previously explored in comparable CA approaches. For instance, the model of Patel et al. (2001) which focuses on acid production does not include oxygen, whilst Gerlee and Anderson's (2007) elegant neural network model abstracts away from specific anaerobic and aerobic waste production in order to study early-growth tumour morphology and genetic inheritance. Instead, we have attempted to implement a similar metabolism to the mean-field simulation approach of Venkatasubramanian et al. (2006) although with some simplifying assumptions. We implement five different cell states - proliferation and quiescence, in either aerobic or anaerobic forms, labelled $p, q$ and $p_{a n}, q_{a n}$, respectively, and cell-death as the final state. Whilst the mean-field model of Venkatasubramanian et al. allowed for the consumption of lactate (as a source of carbon) under low glucose, high oxygen conditions, we only consider acid production during times of hypoxic metabolism and do not permit its subsequent consumption. Additionally, we have relied more on the experimental evidence for glucose and oxygen consumption rates and acid production (as reported in Freyer and Sutherland (1985); Patel et al. (2001)) rather than the stoichiometric approach of Venkatasubramanian et al. (or the site-specific parameter method of Gerlee and Anderson).

Transitions between states for a given site are managed by a state transition algorithm as elaborated in Fig. 3 and follow as close as possible to experimentally $\underset{\text { about }}{\Leftarrow \text { Fig } 3}$

reported phenomena. Specifically, each site responds to the local concentration of here glucose $\left(n_{i}\right)$, oxygen $\left(o_{i}\right)$ and metabolic waste $\left(w_{i}\right)$, implemented directly as hydrogen 
ions in our model. It is known cellular metabolism is significantly affected by local pH (Casciari et al., 1992) and so, the algorithm begins by checking the site pH level, and in accordance with Dairkee et al. (1995), if the $\mathrm{pH}$ is lower than $p H_{\text {death }}=6.0$ the cells at the site undergo death. For our purposes, this is equivalent to necrosis (i.e. unprogrammed cell death) since we have left the inclusion of programmed cell death for further model refinement. Furthermore, as reported in Casciari et al. (1992) if the environmental $\mathrm{pH}$ is smaller than $p H_{c r i t}=6.4$ yet higher than the cell death threshold, the site enters the quiescent state. This leaves all sites with a site $\mathrm{pH}$ greater than 6.4 able to enter one of the proliferative states.

We assume that the most preferred form of proliferation for a site is aerobic proliferation, and thus, at the next decision point, a check of the environmental oxygen concentration $\left(\left[\mathrm{O}_{2}\right]\right)$ is made. High values of oxygen concentration will lead to either aerobic proliferation or quiescence, depending on the environmental glucose concentration $([\mathrm{CHO}])$, whereas hypoxic conditions leads to either anaerobic proliferation or entry into the anaerobic quiescence section of the algorithm for high and low levels of glucose, respectively. It is possible that a site could have high oxygen concentration yet low glucose concentration and subsequently will arrive at the aerobic quiescence state, whereas sites with low environmental oxygen concentration and low glucose concentration will move to either the anaerobic quiescent state (the lowest level of metabolism in our model) or site death (if minimal glucose requirements are not met). The parameters and references for all state transition points, and consumption (production) levels within each state are summarised in Table $1 . \Leftarrow$ Note that some of presented data were not reported in the experimental literature $\begin{gathered}\text { about } \\ \text { here }\end{gathered}$ 
(to our knowledge) and hence some assumptions had to be made, for details see Section 3.

\subsection{Nutrient Transport 85 Replenishment}

Since we are investigating the in vitro world of tumour spheroids, we assume that the tumour mass grows within a vessel much larger than the volume of tumour under study. External sites to the tumour mass are assumed to be replenished with a virtual medium consisting of dissolved concentrations of glucose, oxygen and acid, labelled $n_{e x}, o_{e x}$ and $w_{e x}$, respectively. Experiments conducted by Freyer et al. on spheroids (for details see Freyer (1988) or Freyer and Sutherland (1980)) have shown that concentrations of glucose, oxygen and $\mathrm{H}^{+}$ions in the medium never decreased by more than $5 \%$ of the initial value in fresh medium over the growth period. Hence, we update all sites outside of the tumour mass (those outside of a minimal radius that encompasses all occupied sites) to initial (fresh) medium concentrations each time step.

To account for the diffusion of nutrients $\left(\mathrm{CHO}, \mathrm{O}_{2}\right)$ into the tumour mass and waste products $\left(\mathrm{H}^{+}\right)$out of the tumour mass, at each time-step a numerical diffusion algorithm is applied to an idealised circular area encompassing the tumour completely. Boundary conditions are maintained at the medium concentrations throughout this update procedure. This approach has the added computational benefit of only applying the time-consuming numerical diffusion calculations to a subset of the CA 'world'.

Each diffusion step is achieved by repeated applications of a numerical diffusion 
calculation over all sites in the circular subset:

$$
x_{i}^{t+1}=\frac{\alpha}{f}\left(\sum_{j \in \mathcal{O}^{i}} x_{j}^{t}+\frac{1}{\sqrt{2}} \sum_{j \in \mathcal{D}^{i}} x_{j}^{t}-f x_{i}^{t}\right)+x_{i}^{t},
$$

where $\mathcal{O}^{i}$ is the set of four sites orthogonally adjacent to site $i$ whilst $\mathcal{D}^{i}$ is the remaining four sites diagonally adjacent to site $i$, and $f=4+2 \sqrt{2}$ is the normalising term. The composition of (5) ensures that the diffusion of nutrients occurs isotropically across the D2Q9 lattice (following Wolf-Gladrow (2005))1․ The numerical diffusion coefficient $\alpha$ represents the scaled value of the reported diffusion coefficient from the literature. As in Table 1, we use values reported in Casciari et al. (1988), Venkatasubramanian et al. (2006) and Crone and Levitt (1984) for the CHO, $\mathrm{O}_{2}$ and $\mathrm{H}^{+}$diffusion coefficients, respectively.

\subsection{Cell Division E Dynamics}

As discussed above, the proliferation states (aerobic and anaerobic) imply that cells will add $\Delta t$ to their proliferation register $p_{i}$ each time-step, which counts the total time the site $i$ has spent in the proliferation state. In this way, cells are assumed to always be preparing to divide, and once they have accrued their own cycle-time in the proliferation register, they become candidates to divide.

When a site is filled by cells it is endowed with the same number of cells per site $(N)$ as its parent site along with its own site cycle time chosen from a normally distributed population $\bar{p} \sim N\left(p_{0}, \sigma_{0}\right)$. The normal distribution was chosen for each site's cycle-time for two reasons. First, genetic differences between cells contribute to variation in cycle times that theoretically and experimentally appear to fit this

\footnotetext{
${ }^{1}$ The authors thank an anonymous referee for the suggestion of this methodology.
} 
distribution (Mustafin and Volkov, 1982; Preisler et al., 1995). Second, by sampling cell cycle times from a broad distribution, we overcome a numerical artifact that could arise with constant cycle times, namely, an unnatural 'step' growth phenomenon in bulk tumour characteristics due to all nutrient-rich cell division times being in sync. It should be noted here that an important consequence of quiescence is the slowing of the mitotic rate since each site is endowed with its own cycle time and a 'mitotic register'. This register is only advanced (i.e. by $\Delta t$ ) when a site is in either the aerobic or anaerobic proliferative mode. In all other states, this register remains unchanged.

After time, if a site's proliferation register is equal to or larger than the site's mitotic cycle time, it becomes a candidate for division (see division algorithm presented on Fig. 4). To ensure the legality of the $2 \mathrm{~d}$ CA environment, a prospective division candidate lattice site must have an empty lattice site within its 8 near$\Leftarrow$ Fig 4 neighbours to put the new daughter cells into. This is a more preferable approach to that of Mansury and Deisboeck (2003, 2004); Mansury et al. (2002) who allow the new daughter cell to be placed in the same location as the parent cell (which effectively assumes that cells could be of arbitrarily small size).

Furthermore, in our model, cells cannot move in an active way as it was assumed in Dormann and Deutsch (2002); Mansury and Deisboeck (2003, 2004); Mansury et al. (2002). Instead our approach is similar to that of earlier proposed concepts such as Kansal et al. (2000a,b). In this methodology, a candidate division site goes through a search process beginning with adjacent sites and expanding outwards until an unoccupied lattice site was found. Whilst we restrict the search space to 
only adjacent sites, we retain the implicit contact-inhibition assumption since a site with no free adjacent sites (in the Moore neighbourhood) is not permitted to divide and must wait until a free site becomes available. Such a limited 'search' process is particularly relevant in our many-to-one model since allowing a 'packet' of daughter cells to be transported further than near-neighbour sites would be questionable.

Unfortunately, the simple CA, grid-like setup can produce morphological artifacts due to the repeated application of the division/update rules (see discussion in Drasdo (2005)). We adjust for this phenomenon by applying a novel probabilistic overlay to the placement of daughter sites relative to their parent. Specifically, define by $p$ and $q$ the probabilities of placing a daughter site at one of the orthogonal (i.e. vertical or horizontal) or diagonal positions in the grid, respectively. One can then write the expected speed of advance in the vertical (or horizontal) direction as $e_{x}=p+2 q$ whilst in the diagonal direction we have $e_{x y}=\sqrt{2}(p+q)$. By introducing the relationships $q=\lambda p$ and $p+q=1$ we obtain the ratio of the advance speeds in the following form

$$
\frac{e_{x y}}{e_{x}}=\theta=\frac{\sqrt{2}(1+\lambda)}{2 \lambda+1}
$$

It is clear that the advance speeds in the diagonal and the vertical (horizontal) directions are equal if the condition

$$
\lambda^{*}=\frac{1-\sqrt{2}}{\sqrt{2}-2},
$$

holds. Hence, we set the exact ratio of probabilities $\lambda=\lambda^{*}$ to ensure that the advance speeds are balanced. Note that within this general framework $\lambda=0$ corresponds to a strict Von Neumann (vertical, horizontal only) regime, whilst for 
$\lambda \gg \lambda^{*}$ the diagonal direction is emphasised, creating a square-like limit propagation boundary. This approach is preferable to others mentioned in references such as Drasdo (2005) (e.g. random lattices) since it adjusts for the numerical artifact without detracting from the general grid-like (i.e. matrix) underpinnings of the CA model. The latter is retained in our approach to exploit various native array-based computational functions.

\subsection{Timing}

Model timing in the CA setup follows a discrete set of steps as indicated in Alg. 1. After scaling (due to the user choice of $N$ ), the world is initialised and then the main update loop runs until the end of the specified time. Each update is equivalent to one time step $\Delta t$, and as such, all consumption and production (and diffusion) operations are scaled to occur in per $\Delta t$ time units.

The choice of actual time-step used in the simulations to follow was guided by two principles. First, it is clearly desirable, if at all possible, to use a time-step greater than 1s such that the model requires less updates per total experiment duration (usually set to 19 days). Second, an upper bound is placed on this choice due to the fact that nutrient demands due to occupied sites in the tumour mass must be met by the numerical diffusion algorithm. If the chosen value for $\Delta t$ is too high, nutrients (recall, scaled to per $\Delta t$ amounts) will be consumed by the tumour above the rate of nutrient supply at the site. Calculations can be made after scaling for a given choice of $N$ that yield this cross-over point. In our setup this point occurred at approximately 12s. After a little experimentation (not shown) a $\Delta t$ value of $6 \mathrm{~s}$ 
was chosen to be well within this upper limit.

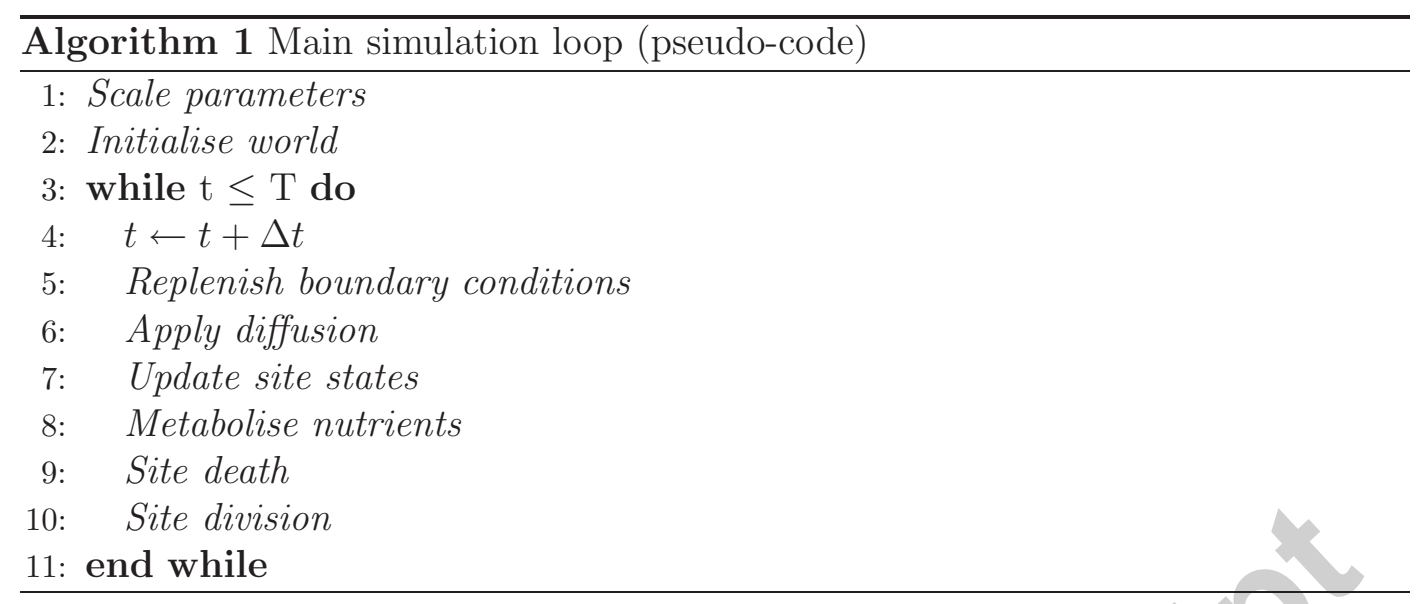

\section{Model Parameters}

As can be seen from Table 1, the model utilises 26 input parameters. However, in almost all cases, owing to the exact scaling relationship due to the choice of $N$ and $\Delta t$, these are not variables as such, but rather represent real data inputs to the modelling environment. For the present work, the EMT6/Ro (mammary sarcoma) cell line was chosen since it has been extensively studied in vitro and thus, data covering nearly the complete set of inputs was found. The reader is referred to the references of Table 1 for further reading.

Note that some data were not found in the literature and in these cases, reasonable estimates were calculated and are shown in the Table 1. For instance, the $\mathrm{CHO}$ consumption rates for quiescent anaerobic sites was estimated by assuming the same ratio of $\mathrm{CHO}$ consumption for proliferative anaerobic to aerobic cells as deduced from the experimental literature. Likewise, we have assumed that necrotic (dead) sites consume neither $\mathrm{O}_{2}$ nor $\mathrm{CHO}$.

In our approach we assume that cells produce different amounts of toxic waste 
depending on the cell state. Namely, following Patel et al. (2001) we assume that proliferating cells performing the aerobic metabolism produce $w_{p}=1.0 \times 10^{-5}{\mathrm{mM} . \mathrm{s}^{-1}}^{-1}$ while, due to the fact that quiescent cells are essentially metabolically inactive, the quiescent calls produce are assumed to produce $w_{q}=5.0 \times 10^{-7} \mathrm{mM} . \mathrm{s}^{-1}$ of hydrogen ions. Cells that proliferate but use the anaerobic metabolism produce much more metabolic waste. Since, during the anaerobic metabolism from one glucose molecule 2 molecules of lactic acid is produce and for each lactate molecule, one hydrogen ion is formed we assume that $w_{p l}=2 C_{p l}$ and $w_{q l}=2 C_{q l}$ mol.(cell.s) ${ }^{-1}$ for low oxygen proliferation and quiescence, respectively. In our model we assume that the necrosis process takes about 30 min and causes that cell material is released directly into the extracellular space. Consequently, we estimate that $w_{n}=180 w_{p l}$ mol.cell ${ }^{-1}$.

Naturally, after calculation of the lattice site side length $u$ (e.g. by setting $N=400)$ units of all consumption/production rates used in our model were converted from mol.(cell.s) $)^{-1}$ or mM.s ${ }^{-1}$ to mol.(site. $\left.\Delta t\right)^{-1}$. Similarly the medium concentrations given in $\mathrm{mM}$ were changed to mol.(site) ${ }^{-1}$.

\section{Results}

\subsection{Model Validation}

To investigate the model, results were prepared for conditions as close as possible to that of the study of EMT6/Ro 3D cultivated spheroids reported in Freyer and Sutherland (1985) (see Table 1 above). An example visualisation of a typical tumour progression over 16 days $\left(n_{e x}=16.5 \mathrm{mM}, o_{e x}=0.28 \mathrm{mM}\right.$ and $\left.p H_{e x}=7.4\right)$ is given in Fig. 5 showing the progression from initial seed to a grown tumour mass undergoing $\Leftarrow$ Fig 5 
central necrosis (white areas in middle of tumour). As expected, after a short time, most sites exist in a quiescent state, with only peripheral sites capable of a proliferative metabolism. The later stage tumour morphology displays a familiar, roughly circular geometry with scattered regions of necrosis evident in low densities throughout the tumour mass in addition to the larger necrotic region at the centre. These features are similar to the morphologies reported in comparable CA simulation models (Gerlee and Anderson, 2007, see Fig. 7, p.594) and (Dormann and Deutsch, 2002, see Fig. 8), mean-field numerical approaches (Venkatasubramanian et al., 2006, see Fig. 7, p.447) and experimental histologies (Walenta et al., 2000; Yu et al., 2007).

To validate the expected behaviour of the many-to-one scaling approach as described in Section 2.2 a series of experiments was conducted under the same conditions as reported above (with $n_{e x}=16.5 \mathrm{mM}, o_{e x}=2.8 \mathrm{mM}$ and $p H_{e x}=7.4$ ) except for the value of $N$ which were varied in the set $\{10,100,400\}$. Results from this study (mean of two trials at each condition) are presented in Fig. 6 and show a good agreement with the predictions of the theory with all rescaled cell counts falling on top of each other in a linear manner as predicted by Eq. 3 despite the value of $N$ differing over more than two orders of magnitude.

Next, a series of experiments was conducted at a range of $\mathrm{CHO}$ and $\mathrm{O}_{2}$ medium concentrations as indicated in Table 2 to compare the model outputs with those of $\Leftarrow$ Tab 2 about Freyer and Sutherland (1985) and Freyer and Sutherland (1986). To obtain quan- here titative results, five distinct trials were run for $2.736 \times 10^{5}$ (6s equivalent) updates, representing the study time of 19 days. A single 19 day trial took approximately 35 min of CPU time to complete ${ }^{2}$.

\footnotetext{
${ }^{2}$ Data obtained in the MATLAB mathematical programming language on an Apple MacPro (2
} 
Data were taken on various aspects of the tumour's progression including viable tumour diameter, necrotic core diameter (if any), cell nutrient consumption and cell state (either proliferating or quiescent) and position. The diameter was calculated in an analogous way to that reported in Freyer and Sutherland (1980) (p.3957) by taking the geometric mean of 2 orthogonal measurements from the position of occupied sites. Similarly, cell count (i.e. number of filled sites multiplied by $N$ ) and tumour volume $\left(V_{\text {tumour }}=u \pi R^{2}\right)$ of the spreading tumour mass was calculated after scaling conversion.

Fig. 7 shows the bulk tumour growth dynamics for a selection of these experiments. Plot (a) shows the linear relationship obtained under $n_{e x}=16.5 \mathrm{mM}$ and $o_{e x}=0.28 \mathrm{mM}$ (other plots were also linear up to the point where the necrotic portions of the model tumours disrupted the measurement of tumour diameter), and plot (b) indicates the total cell count for fixed $o_{e x}=0.28 \mathrm{mM}$ and varying medium concentration of CHO. Gompertz model $\left(C(t)=C_{0} \exp (A / B(1-\exp (-B t)))\right.$ lines of best fit were fitted by the standard MATLAB non-linear fminsearch procedure to yield standard saturation counts and initial doubling time data. Complete summary results from these experiments are reported in Table 2.

The results from these experiments indicate that the model has reasonable to very good agreement with the experimental values as reported in the target study of Freyer and Sutherland (1985). The reference focuses on medium conditions of $n_{e x}=5.5 \mathrm{mM}, o_{e x}=0.28 \mathrm{mM}$ and $\mathrm{pH}$ level equal 7.4 and the results of this study (where reported) are reproduced in the last column of numbers in the summary table for ease of comparison. One can see that for similar medium concentrations (the $\bar{x}$ Quad-Core Intel Xenon chipset, 8MB RAM) running Mac OS X 10.5.6. 
central column of model values under medium $\left[\mathrm{O}_{2}\right]=0.28 \mathrm{mM}$ ) many model outputs are on par with the reference. All comparable values are found to be within the same order of magnitude as the ref. and in some cases (e.g. diameter at onset of necrosis) the model returns a remarkably similar absolute value to the ref. There are some discrepancies with the ref., for example, the consumption data taken from the model are somewhat different to those reported in Freyer and Sutherland (1985) (Table 1, 2, p.520) however, it is to be noted that the ref. reports on separate experiments conducted on single exponential cells exposed to the various conditions, rather than taking data (as we do) from the actual spheroids themselves. Indeed, it remains for the updated approaches such as those used with alternate cell lines (Rat1-T1, MR1) (Walenta et al., 2000) to yield spheroid-applicable data of this nature.

\subsection{The Onset of Necrosis}

In addition to the 1985 study of Freyer and Sutherland (1985), the EMT6/Ro cell line was the subject of another report by the authors which specifically considered the onset and progression of necrosis in tumour spheroids cultivated at a variety of medium $n_{e x}$ and $o_{e x}$ values and thus provides an important set of comparisons for the present work. As in Freyer and Sutherland (1985), our model produces little variation in tumour (growth, necrosis) characteristics when the value of $o_{e x}$ is varied at a given value of $n_{e x}$ (see Table 2). Hence, we focus our comments on comparisons of behaviour due to variations in $n_{e x}$ at a given value of $o_{e x}=0.28 \mathrm{mM}$.

Typical morphologies at the onset of necrosis for the three $n_{e x}$ conditions are presented in Fig. 8. As expected, and in line with the data presented in Table 3 
(p.3508) of Freyer and Sutherland (1986) the diameter of the tumour mass at the onset of necrosis is proportional to the value of $n_{e x}$. In other respects, the tumours show similar morphological characteristics such as a large area of anaerobic necrotic material in the centre of the tumour, surrounded by a thin proliferative boundary.

Confirmation of these final characteristics is provided by Fig. 9 which shows the proportion of sites/cells in each of the four possible states for the largest of the tumours 'grown' at $n_{e x}=16.5 \mathrm{mM}$. It is clear that after an initial induction period that exists up to approximately $500 \mu \mathrm{m}$ where proliferative sites far outnumber quiescent sites, these sites switch very rapidly into an anaerobic quiescent mode, if away from the boundary, or anaerobic proliferative state if at the boundary itself. These data are very similar to those presented for $n_{e x}=5.5 \mathrm{mM}\left(o_{e x}=0.28 \mathrm{mM}\right)$ in Fig. 6 of Freyer and Sutherland (1985) where $G_{1}$ phase cells account for greater than $80 \%$ of the cells in spheroids at late growth times as in our data. It is to be noted that the switch to quiescence identified in our model is not due to 'contact inhibition' considerations, recalling that site states are set independent of whether neighbouring sites are free for daughter cells to be placed. We can thus only conclude that the overwhelming presence of quiescent sites at later time periods in our model is due to unfavourable environmental nutrient and waste $\left(\mathrm{H}^{+}\right)$conditions.

Indeed, these conditions were directly analysed utilising a methodology similar to that of Walenta et al. (2000) where central ATP, glucose, $\mathrm{O}_{2}$ and lactate concentrations were obtained for Rat1-T1 and MR1 spheroids via bioluminescence imaging. Comparative data presented in Table 2 and Fig. 10 were prepared by averaging the central tumour region (approx. $300 \times 300 \mu \mathrm{m}$ ) concentrations of each environmental 
constituent in the model for $n_{e x}=16.5 \mathrm{mM}$ during the tumour growth progression. To aid comparison, the data have been normalised to a value of 1 for the external concentrations, whilst 0 is equivalent to minimum concentrations. As a guide, the final central concentrations of $\mathrm{CHO}$ and $\mathrm{O}_{2}$ were 1.101 and $0.158 \mathrm{mM}$, respectively (see Table 2), whilst the mean final central $\mathrm{pH}$ was 6.37 .

It would appear from this plot that the onset of necrosis in our model was caused by either the reduction in availability of $\mathrm{CHO}$, or the slight decrease in $\mathrm{pH}$ values evident just before the onset of necrosis occurred. However, a compilation of the average values of central $[\mathrm{CHO}],\left[\mathrm{O}_{2}\right]$ and $\mathrm{pH}$ at the onset of necrosis itself from across the different $n_{e x}$ study conditions (see Table 3) confirms that minimal metabolism $\Leftarrow$ (i.e. anaerobic quiescence) requirements were met at the point of necrosis for $\mathrm{O}_{2}$ as well as $\mathrm{pH}$ level, with $[\mathrm{CHO}]$ being the only sub-minimal requirement. These data again confirm that the production of toxic waste was not high enough in the middle of the tumour to cause cell death outright, and indeed, since most internal tumour sites quickly switch to an anaerobic quiescent metabolism (refer Fig. 9), the production of $\mathrm{H}^{+}$in the centre of the tumour was negligible. This feature of the metabolism dynamics is clearly supported by the rates of consumption (production) for CHO, $\mathrm{O}_{2}\left(\right.$ and $\left.\mathrm{H}^{+}\right)$as presented in Fig. 11. Comparison with similar data presented for Rat1-T1 and MR1 found in Fig. 6 (p.517) of Walenta et al. (2000) and for the theoretical predictions of $\mathrm{O}_{2}$ consumption presented in Groebe and Mueller-Klieser (1996) shows that the model produces very faithful bulk metabolic dynamics, with the initial rise and subsequent fall of glucose consumption and lactate $\left(\mathrm{H}^{+}\right)$production with tumour diameter growth faithfully reproduced. These data are somewhat 
expected from an analysis of the site state population fractions (see Fig. 9) as the early presence of proliferation (high $\mathrm{H}^{+}$output, and glucose consumption) is followed by the transition to anaerobic quiescence (low oxygen and glucose demands and negligible $\mathrm{H}^{+}$production). Clearly, the major portion of the central tumour produces little $\mathrm{H}^{+}$waste in the lead-up to necrosis.

\subsection{The Progression of Necrosis}

Following initiation, the progression of necrosis under various medium $\mathrm{CHO}$ concentrations in the model follows closely to that reported in Freyer and Sutherland (1986). Since necrosis appeared in the $n_{e x}=16.5 \mathrm{mM}$ study only at very late time periods, a longer (25 day) data set (again with five repeats) was prepared for just this medium concentration to generate necrosis activity of comparable magnitude for comparison to the lower medium CHO concentrations. The joint-data from these studies are presented in Figs. 12, 13 and 14. In accordance with Freyer and Sutherland (1986) across comparable conditions, we find that the viable cell rim decreases with tumour growth progression (see Fig. 12). Indeed, these data are consistent with those presented earlier which suggested a strong link between excessive declines in site $\mathrm{CHO}$ concentration and the onset of necrotic activity.

Further evidence is presented in Fig. 13 which compares the average thickness of the viable cell rim after the onset of necrosis for different levels of $n_{e x}$. For ease of comparison, the approximations to the data reported in Fig. 4 of Freyer and Sutherland (1986) are also presented in the figure (filled markers). Whilst the model generates very good approximations to the experimental data for the 
lower concentrations $(2.8 \mathrm{mM}$ and $5.5 \mathrm{mM})$, the $16.5 \mathrm{mM}$ case departs somewhat. Nevertheless, it is clear that as in Freyer and Sutherland (1986) the viable sites in our model are likewise sensitive to the external glucose concentration, if not more sensitive, all else being equal. Finally, Fig. 14 completes this line of inquiry, showing comparable data to that reported for $n_{e x}=5.5 \mathrm{mM}$ in Table 3 (p. 520) of Freyer and Sutherland (1985), again filled markers are presented for the data from Freyer and Sutherland (1985) for comparison. Here, as in Freyer and Sutherland (1985) the necrotic volume fraction progresses with a positive, linear gradient following the onset of necrosis.

\section{Discussion}

There are two possible explanations in our model for the evidence on necrosis presented above. One explanation could be that after a certain time, the concentration of glucose drops below critical levels for viable metabolism, and this drop arrives almost simultaneously across a large portion of the central tumour mass. Alternatively, as is advanced by Freyer and Sutherland (1986), the progression of necrosis could be due to a two-step process where first, a combination of factors (e.g. environmental concentrations, site-pressure) causes a dense region at the centre of the tumour to undergo necrosis, and then, these necrotic events cause the release of a large quantity of waste materials which immediately cause the death of surrounding cells, already under stress due to unfavourable environmental conditions.

The evidence presented would appear to find in favour of the former (sub-minimal metabolism requirements), and against the latter (death via indirect toxicity). Al- 
though necrosis advances rapidly in our model immediately following the onset of necrosis (Fig. 14) as was similarly reported in Freyer and Sutherland (1985), like Walenta et al. (2000), we do not observe a coincident spike in waste $\left(\mathrm{H}^{+}\right)$concentrations (refer Fig. 11) despite allowing for a one-off production of 30min worth of $\mathrm{H}^{+}$at any given necrotic site. Furthermore, both leading up to the onset of necrosis, and afterwards (refer Table 2 and 3) the central pH never appears to fall below 6.0, our effective $\mathrm{pH}$ floor to any viable metabolism.

Furthermore, although not shown, the present setup is able to produce a necrotic core in identical environments to those studied above, but without the waste $\left(\mathrm{H}^{+}\right)$ module present in the model (data not shown). Hence, at present, the model appears to suggest a purely sub-minimal nutrient environment rationale for the onset and spread of necrosis. This finding stands in contrast to that of both Walenta et al. (2000) and Freyer and Sutherland (1986) who both (for different reasons) rule out the nutrient deficiency argument. Nevertheless, the model is in agreement with the detailed data of Walenta et al. (2000) in that it shows that central $\mathrm{H}^{+}$concentrations are not high enough (under the present specification) to cause necrosis. Indeed, it can be seen in Table 2 that the onset of necrosis under each medium condition occurs at a diameter some $25 \%$ to $60 \%$ in excess of that reported by equivalent studies in Freyer and Sutherland (1986). This further supports the theory that normal tumours do not reach this level of nutrient depletion before necrosis, and instead, another process causes unprogrammed cell death in advance of this nutrient-depletion point.

Thus, the analysis suggests several important conclusions and suggestions for further work to the field. First, assuming that the present model is an accurate ab- 
straction of tumour spheroid dynamics (criticisms of the present model are addressed below) the model requires more accurate input parameters than are presently available in the experimental literature. In particular, the cellular production rates of acid under the different regimes in general, and the anaerobic quiescent regime in particular, appear critical to gaining further insight. Second, if one assumes that the present results are not greatly changed by the utilisation of more accurate parameters (noting the model's encouraging accuracy in many areas of known spheroid behaviour), the model has shown that there exists an as yet unknown 'X-factor' that causes necrosis to occur, despite favourable metabolic and $\mathrm{pH}$ conditions prevailing. Of course, suggestions for this factor already lie in the model of Freyer and Sutherland (1986) who specified a general 'waste' term, rather than $\mathrm{H}^{+}$in particular. It is quite possible that other forms of waste (e.g. cellular components) are a more potent source of toxicity to the central tumour region. Freyer's striking 1988 experiments of tumour growth suppression when inner-spheroid necrotic liquid was present in the medium are still the best support for this hypothesis. Finally, it also remains for further experimental analysis to determine the adequacy of the peripheral mitotic dynamics theory which results from the CA approach to the computational simulation of tumour growth. Whilst we believe the results presented in this work provide strong evidence for the efficacy of the CA modelling approach, it would be of interest to know if real data, plotted appropriately, also conforms to the theoretical predictions of the model.

Additionally, the continuous PDE work of Ward and King (1997) and Ward and King (1999) provides interesting parallels to the present work. In these studies, live 
and dead cellular material are modelled as a continuum of cells with live cell division and death causing expansion and contraction of the tumour respectively. In contrast to our approach, the loss of cells in these contributions cause a cellular velocity gradient. Of particular interest is Ward and King (1999) which incorporates the assumption of the present work, that ultimately after cell death, the entire cellular volume disappears. In Ward and King (1999) two mechanisms achieve this: first, it is assumed that cell death releases useful components for live cells to metabolise; and second, as in our model, these components may diffuse out of the the tumour mass and thus be lost through leakage. Numerical data and surrounding text presented in Ward and King (1999), Fig. 1 and Fig. 6, provide interesting intuition for observed viable tumour thickness as tabulated in Freyer and Sutherland (1985) and shown in Fig. 12 in the present work.

Ward and King find that after an initial transient phase of exponential expansion in spheroid radius, $S$, growth stabilises to a linear regime due to quiescence of the core. After a time, the necrotic core develops which grows at a faster rate than the linear spheroid radius, thus causing the thickness of the viable rim to quickly reduce initially, before stabilising at a relatively constant thickness. It is also found that under certain parametrisations (high mitotic consumption of cell death components, and high diffusion of the same), a realistic three-layer structure is observed, and that the tumour may obtain the experimentally observed growth saturation limit rather than the travelling-wave (unbounded) limit. Both of these observations (three-layer structure, growth saturation) are replicated in the present model as endogenous outcomes of the modelling setup, although these occur without 
the addition of growth inhibition (data not shown). Clearly further work is needed to probe the competing theories of growth inhibition or mitotic provision due to cellular death in the tumour core. Nevertheless, we find agreement with the authors who state in the Discussion section of Ward and King (1999) that whilst there has been much study of the diffusion of components into the tumour mass, there has been relatively little study of the reverse process: that of components diffusing out of the tumour mass.

There are many attributes of the present setup that could be improved in future versions, and it remains to be seen if such advances bring the in silico results more into line with the in vitro approaches. As mentioned above, the production rates of $\mathrm{H}^{+}$are an obvious point of criticism. Second, it is possible that the abstraction away from formal buffering models for the $\mathrm{H}^{+}$clearance are not adequate. Although our assumption was motivated by similar approaches (e.g. Patel et al. (2001)), since it appears that the production and clearance of $\mathrm{H}^{+}$is critical to the findings of the model, this aspect may need to be revisited. Finally, the model makes no delineation between programmed cell death (apoptosis) and necrosis. Since it is known that ATP is required for successful apoptosis, the prior programmed death of some cells may change both the acidic and glucose environment for those that remain. Although, intuition would suggest that such a complication would further deplete the available glucose and similarly reduce the number of one-off $\mathrm{H}^{+}$producing necrotic events, hence encouraging similar results to that of the present setup.

Another aspect of the model that requires further investigation is the crosssectional nutrient profile. It was observed during all simulations that up until the 
onset of necrosis (figures not shown), the central nutrient concentration declined roughly linearly with tumour diameter. This is in accordance with results obtained by Walenta et al. who used bioluminescence imaging to identify central oxygen and ATP concentrations with spheroid diameter (Walenta et al., 2000). However, this work showed that after the onset of necrosis, central nutrient concentrations stay very low throughout further spheroid growth. This result was also found in Ward and King's PDE model mentioned above (see Fig.12). In contrast, an analysis of the same in our model showed that the central nutrient concentration began to increase post necrosis onset, causing a tri-modal cross-sectional nutrient profile to be observed after time. Whilst the central peak was never enough to sustain a viable cell, it does indicate that some unmodelled phenomena is present in vitro that either prevents nutrients diffusing into the necrotic core, or causes their consumption.

Nevertheless, the approach analysed above offers exciting possibilities for future advances in computational oncology. It is our contention that the many-to-one scaling assumption used as the core of this paper allows for a much wider range of tumour development scenarios to be studied with relatively accurate outputs on many experimental dimensions.

\section{Acknowledgements}

The authors wish to acknowledge the help of Dr. Kai Masur, of Institute of Immunology, University of Witten/Herdecke for helpful comments regarding the details of cellular metabolism. This work was partially supported by the Santa Fe Institute through NSF Grant No. 0200500 entitled "A Broad Research Program 
in the Sciences of Complexity." This paper was prepared within the framework of the EU project "Modelling, Mathematical Methods and Computer Simulation of Tumour Growth and Therapy", MRTN - CT - 2004 - 503661.

\section{REFERENCES}

Andersen, L. K. and Mackey, M. C. (2001). Resonance in periodic chemotherapy: A case study of acute myelogenous leukemia. J. Theor. Biol., 209:113-130.

Bellomo, N. and Forni, G. (1994). Dynamics of tumour interaction with the host immune system. Math. Comp. Model., 20:107-122.

Bodnar, M. and Forys, U. (2005). Time delay in necrotic core formation. Math. Biosci. \& Eng., 2:461-472.

Byrne, H. M. and Chaplain, M. A. J. (1995). Growth of non-nerotic tumours in the presence and absence of inhibitors. Math. Biosci., 130:151-181.

Byrne, H. M. and Chaplain, M. A. J. (1996b). On the role of cell-cell adhesion in models for solid tumour growth. Math. Comp. Model., 24:1-17.

Casciari, J. J., Sotirchos, S. V., and Sutherland, R. M. (1988). Glucose diffusivity in multicellular tumor spheroids. Cancer Res., 48:3905-3909.

Casciari, J. J., Sotirchos, S. V., and Sutherland, R. M. (1992). Variation in tumor growth rates and metabolism with oxygen concentration, glucose concentration, and extracellular pH. J. Cell Physiol., 151:386-394.

Chaplain, M. A. J. (1996). Avascular growth, angiogenesis and vascular growth in 
solid tumours: The mathematical modelling of the stages of tumour development. Math. Comp. Model., 23:47-87.

Chaplain, M. A. J., Ganesh, M., and Graham, I. G. (2001). Spatio-temporal pattern formation on spherical surfaces: Numerical simulation and application to solid tumour growth. J. Math. Biol., 42:387-423.

Crone, C. and Levitt, D. G. (1984). Capillary permeability to small solutes. In Handbook of Physiology: A critical, comprehensive presentation of physiological knowledge and concepts, Section 2: The Cardiovascular System, Vol. IV: Microcirculation, Part 1 (Renkin, E. M. and Michel, C. C., eds).p.414 and pp. 434-437. Bethesda, ML: American Physiological Society.

Dairkee, S. H., Deng, S. H., Stampfer, M. R., Waldman, R. M. and Smith H. S. (1995). Selective cell culture of primary breast cancer. Cancer Res., 35:25162519.

Dormann, S., and Deutsch, A. (2002). Modeling of self-organized avascular tumor growth with a hybrid cellular automaton. In Silico Biology, 2:0035.

Drasdo, D. (2005), Coarse graining in simulated cell populations, Adv. Comp. Sys., 8:319-363.

Ferlay, J., Autier, P., Boniol, M., Heanue, M., Colombet, M., and P, B. (2007). Estimates of the cancer incidence and mortality in europe in 2006. Annals of Oncology, 18:581-592. 
Freyer, J. P. (1988). Role of necrosis in regulating the growth saturation of multicellular spheroids. Cancer Res., 48:2432-2439.

Freyer, J. P. and Sutherland, R. M. (1980). Selective dissociation and characterization of cells from different regions of multicell tumour spheroids. Cancer Res., 40:3956-3965.

Freyer, J. P. and Sutherland, R. M. (1985). A reduction in the in situ rates of oxygen and glucose consumption of cells in emt6/ro spheroids during growth. $J$. Cell. Phys., 124:516-524.

Freyer, J. P. and Sutherland, R. M. (1986). Regulation of growth saturation and development of necrosis in emt6/ro multicellular spheroids by the glucose and oxygen supply. Cancer Res., 46:3504-3512.

Gatenby, R. A., Vincent, T. L., and Gillies, R. J. (2005). Evolutionary dynamics in carcinogenesis. Math. Mod. Meth. Appl. Sci., 15:1619-1638.

Gerlee, P. and Anderson, A. R. A. (2007), An evolutionary hybrid cellular automaton model of solid tumour growth, J. Theor. Bio., 246:583 - 603 .

Groebe, K., and Mueller-Klieser, W. (1996). On the relation between size of necrosis and diameter of tumor spheroids, Int. J. Radiat. Oncol. Biol. Phys., 34(2):395-401.

Hatzikirou, H., Deutsch, A., Schaller, C., and Simon, M. (2005). Mathematical modelling of glioblastoma tumour development: A review. Math. Mod. Meth. Appl. Sci., 15:1779-1794. 
Jiang, Y., Pjesivac-Grbovic, J., Cantrell, C., and Freyer, J. P. (2005). A multiscale model for avascular tumor growth. Biophys. J., 89:3884-3894.

Kansal, A. R., Torquato, S., Chioccaeb, E. A., and Deisboeck, T. S. (2000a). Emergence of a subpopulation in a computational model of tumor growth. J. Theor. Biol., 207:431-441.

Kansal, A. R., Torquato, S., Harsh Iva, G. R., Chioccaeb, E. A., and Deisboeck, T. S. (2000b). Simulated brain tumor growth dynamics using a three-dimensional cellular automaton. J. Theor. Biol., 203:367-382.

Kardar, M. (2007). Statistical Physics of Fields. Cambridge Press.

Mansury, Y. and Deisboeck, T. S. (2003). The impact of 'search precision' in an agent-based tumour model. J. Theor. Biol., 224:325-337.

Mansury, Y. and Deisboeck, T. S. (2004). Simulating ‘structure-function' patterns of malignant brain tumours. Physica A, 331:219-232.

Mansury, Y., Kimura, M., Loboz, J., and Deisboeck, T. S. (2002). Emerging patterns in tumor systems: Simulating the dynamics of multicellular clusters with an agentbased spatial agglomeration model. J. Theor. Biol., 219:343-370.

Moore, H. and Lib, N. K. (2004). A mathematical model for chronic myelogenous leukemia (cml) and t cell interaction. J. Theor. Biol., 227:513-523.

Mustafin, A. T. and Volkov, E. I. (1982). On the distribution of cell cycle generation times. Biosystems, 15(2):111-126. 
Patel, A. A., Gawlinski, E. T., Lemieux, S. K. and Gatenby, R. A. (2001). A cellular Automaton model of early tumor growth and invasion: The effects of native tissue vascularity and increased anaerobic tumour metabolism. J. Theor. Biol., 213:315331.

Piotrowska, M. J. (2008). Hopf bifurcation in a solid avascular tumour growth model with two discrete delays. Math. Comp. Model., 47:597-603.

Piotrowska, M. J. and Foryś, U. (2004). Time delays in avascular tumour growth. In Proceedings of the Tenth National Conference on Application of Mathematics in Biology and Medicine, Świety Krzyż.

Preisler, H. D., Raza, A., Gopal, V., Ahmad, S. and Bokhari, J. (1995). Distribution of cell cycle times amongst the leukemia cells within individual patients with acute myelogenous leukemia. Leukemia Research, 19:693-698.

Preziosi, L (ed) and references therein (2003). Cancer modeling and simulation. Chapman \& Hall/CRC.

Venkatasubramanian, R., Henson, M. A. and Forbes, N. S. (2006). Incorporating energy metabolism into a growth model of multicellular tumor spheroids. J. Theor. Biol., Sept,242(2):440-453.

Walenta, S and Doetsch, J and Mueller-Klieser, W and Kunz-Schughart, L A (2000). Metabolic imaging in multicellular spheroids of oncogene-transfected fibroblasts. J. Histochem Cytochem, Apr, 48(4):509-22. 
Ward, J P and King, J R (1997). Mathematical modelling of avascular-tumour growth. IMA Journal of Mathematics Applied in Medicine $\&$ Biology, 14:39-69.

Ward, J P and King, J R (1999). Mathematical modelling of avascular-tumour growth II: Modelling growth saturation. IMA Journal of Mathematics Applied in Medicine \& Biology, 16:171-211.

Weaver, V. M., Petersen, O. W., Wang, F., Larabell, C. A., Briand, P., Damsky, C., and Bissell, M. J. (1997). Reversion of the malignant phenotype of human breast cells in three-dimensional culture and in vivo by integrin blocking antibodies. $J$ Cell Biol., 137(1):231-245.

Wolf-Gladrow, D. A., (2005). Lattice-Gas Cellular Automata and Lattice Boltzmann Models - An Introduction. Springer.

Yu, P., Mustata, G., and Nolte, D. D. (2007). 3-d video holography through biological tissue. available on the web at http://www.vet.purdue.edu/cristal/ oci-info.htm. 


\section{TABLES}

Table 1 Summary of control parameters (with references) used in the simualations.

\begin{tabular}{|c|c|c|c|c|}
\hline$\overline{\overline{\text { Description }}}$ & Symbol & Value & $\overline{\text { Units }}$ & Ref \\
\hline Cell packing density & $\overline{\rho \rho}$ & $4 \times 10^{8}$ & cell.cm ${ }^{-3}$ & FS1985 \\
\hline $\begin{array}{l}\text { Cell cycle time mean (standard de- } \\
\text { viation) }\end{array}$ & $p_{0}\left(\sigma_{0}\right)$ & $22(1)$ & $\mathrm{h}$ & $\begin{array}{l}\text { FS1980, } \\
\text { FS1985, } \\
\text { JG2005 }\end{array}$ \\
\hline Number of cells per site & $N$ & 100 & cell.site ${ }^{-1}$ & set \\
\hline Unit side-length & $u$ & 100 & $\mu \mathrm{m}$ & calc. \\
\hline Update $\mathrm{t}$ & $\Delta t$ & 6 & $\mathrm{~s}$ & set \\
\hline Critical pH: prolif. $\rightarrow$ quiesc. & $p H_{\text {crit }}$ & 6.4 & & CS1992 \\
\hline Critical pH: quiesc. $\rightarrow$ death & $p H_{\text {death }}$ & 6.0 & & DK1995 \\
\hline \multicolumn{5}{|l|}{ Medium } \\
\hline Medium [CHO] & $n_{e x}$ & $2.8-16.5$ & $\mathrm{mM}$ & FS1985 \\
\hline Medium $\left[\mathrm{O}_{2}\right]$ conc. & $o_{e x}$ & $0.035-0.280$ & $\mathrm{mM}$ & FS1985 \\
\hline Medium pH level & $p H_{e x}$ & 7.4 & & FS1985 \\
\hline Diffusion coef. & & 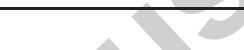 & & \\
\hline CHO diffusion coef. & $\overline{D_{n}}$ & $9.1 \times 10^{-5}$ & $\mathrm{~cm}^{2} \cdot \mathrm{s}^{-1}$ & CS1988 \\
\hline $\mathrm{O}_{2}$ diffusion coef. & $D_{o}$ & $1.82 \times 10^{-5}$ & $\mathrm{~cm}^{2} \cdot \mathrm{s}^{-1}$ & VK2006 \\
\hline $\mathrm{H}^{+}$diffusion coef. & $D_{w}$ & $1.1 \times 10^{-5}$ & $\mathrm{~cm}^{2} \cdot \mathrm{s}^{-1}$ & CL1984 \\
\hline \multicolumn{5}{|l|}{ Proliferating Cells } \\
\hline Aerob. prolif. CHO cons. rate & $C_{p}$ & $18 \times 10^{-17}$ & mol.(cell.s) $)^{-1}$ & FS1985 \\
\hline Anaerob. prolif. $\mathrm{CHO}$ cons. rate & $C_{p l}$ & $52 \times 10^{-17}$ & mol.(cell.s $)^{-1}$ & FS1985 \\
\hline Aerob. prolif. $\mathrm{O}_{2}$ cons. rate & $O_{p}$ & $8.3 \times 10^{-17}$ & mol.(cell.s $)^{-1}$ & FS1985 \\
\hline Anaerob. prolif. $\mathrm{O}_{2}$ cons. rate & $O_{p l}$ & 0 & mol.(cell.s $)^{-1}$ & FS1985 \\
\hline Aerob. prolif. $\mathrm{H}^{+}$product. rate & $w_{p}$ & $1 \times 10^{-5}$ & mM.(s) ${ }^{-1}$ & PT2001 \\
\hline Anaerob. prolif. $\mathrm{H}^{+}$product. rate & $w_{p l}=2 C_{p l}$ & $104 \times 10^{-17}$ & mol.(cell.s $)^{-1}$ & est. \\
\hline \multicolumn{5}{|l|}{ Quiescent Cells } \\
\hline Aerob. quiesc. CHO cons. rate & $\overline{C_{q}}$ & $15 \times 10^{-17}$ & mol.(cell.s $)^{-1}$ & FS1985 \\
\hline Anaerob. quies. $\mathrm{CHO}$ cons. rate & $C_{q l}=\frac{C_{p l}}{C_{p}} C_{q}$ & $43 \times 10^{-17}$ & mol.(cell.s $)^{-1}$ & $\begin{array}{l}\text { est., } \\
\text { FS1985 }\end{array}$ \\
\hline Aerob. quiesc. $\mathrm{O}_{2}$ cons. rate & $O_{q}$ & $5.5 \times 10^{-17}$ & mol.(cell.s $)^{-1}$ & FS1985 \\
\hline Anaerob. quiesc. $\mathrm{O}_{2}$ cons. rate & $O_{q l}$ & 0 & mol.(cell.s $)^{-1}$ & FS1985 \\
\hline Aerob. quiesc. $\mathrm{H}^{+}$product. rate & $w_{q}$ & $0.05 \times 10^{-5}$ & $\mathrm{mM} \cdot(\mathrm{s})^{-1}$ & PT2001 \\
\hline Anaerob. quiesc. $\mathrm{H}^{+}$product. rate & $w_{q l}=2 C_{q l}$ & $86 \times 10^{-17}$ & mol.(cell.s) $)^{-1}$ & est. \\
\hline \multicolumn{5}{|l|}{ Dead Cells } \\
\hline Dead cells CHO cons. rate & $C_{\text {death }}$ & 0 & mol.(cell.s) $)^{-1}$ & ass. \\
\hline Dead cells $\mathrm{O}_{2}$ cons. rate & $O_{\text {death }}$ & 0 & mol.(cell.s $)^{-1}$ & ass. \\
\hline Necrotic material $\mathrm{H}^{+}$product. rate & $w_{n}$ & $9.0 \times 10^{-4}$ & mM.(site $)^{-1}$ & est. \\
\hline
\end{tabular}

Notes

FS1985, Freyer and Sutherland (1985); FS1980, Freyer and Sutherland (1980); JG2005, Jiang et al. (2005); CS1992, Casciari et al. (1992); CS1988, Casciari et al. (1988); DK1995, Dairkee et al. (1995); VK2006, Venkatasubramanian et al. (2006); CL1984, Crone and Levitt (1984); PT2001, Patel et al. (2001) 
ACCEPTED MANUSCRIPT

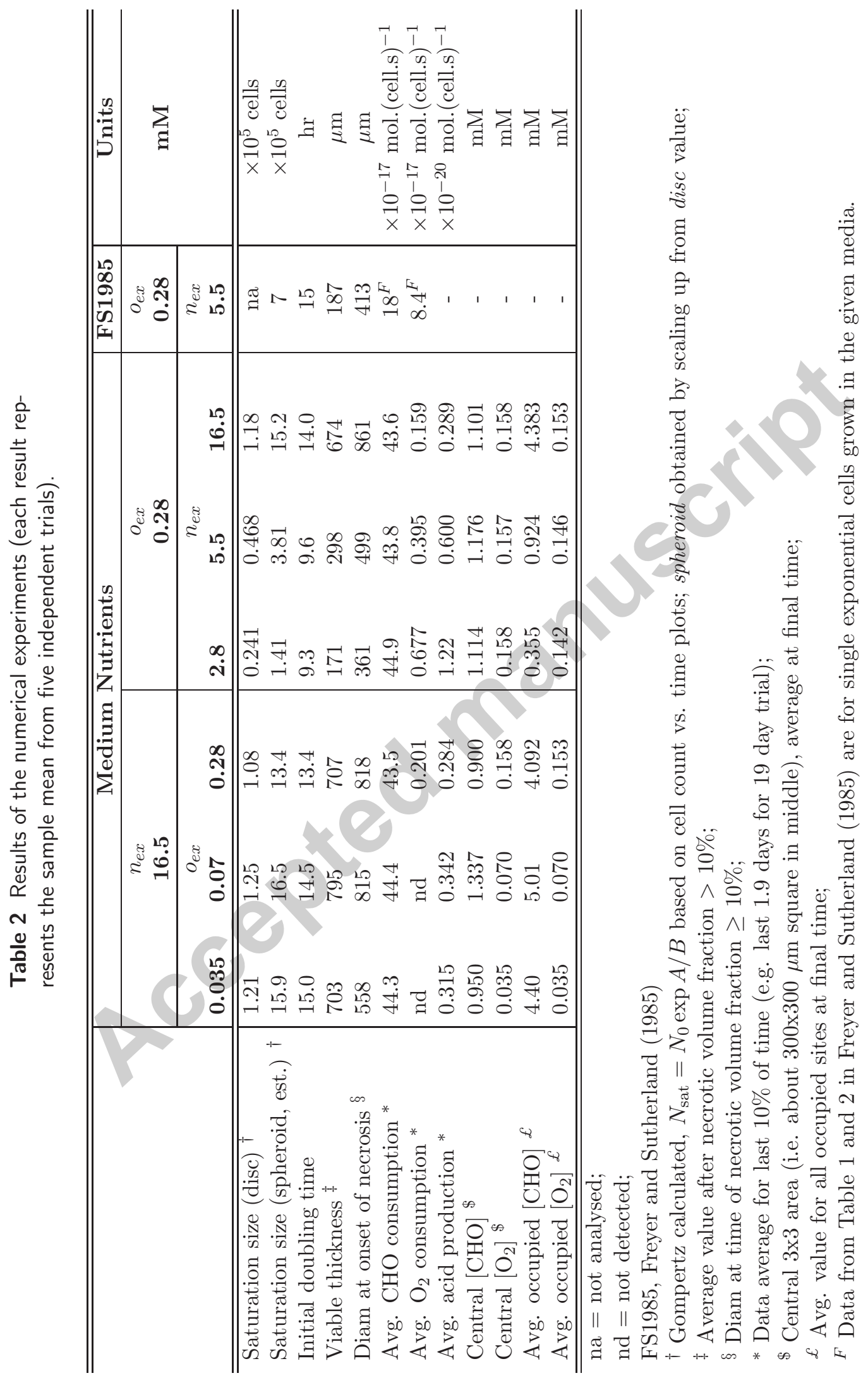


Table 3 Average central concentrations of $\mathrm{CHO}$ and $\mathrm{O}_{2}$, and $\mathrm{pH}$, at the onset of necrosis compared to critical values for minimal metabolism.

\begin{tabular}{l|ccc|c}
\hline \hline & \multicolumn{2}{|c|}{$o_{e x}=\mathbf{0 . 2 8}(\mathbf{m M})$} & Critical \\
\cline { 2 - 4 } & \multicolumn{3}{|c|}{$n_{e x}(\mathbf{m M})$} & value \\
& $\mathbf{2 . 8}$ & $\mathbf{5 . 5}$ & $\mathbf{1 6 . 5}$ & \\
\hline \hline Central $[\mathbf{C H O}](\mathbf{m M})$ & 0.680 & 0.539 & 0.713 & 1.04 \\
Central $\left[\mathbf{O}_{2}\right](\mathbf{m M})$ & 0.152 & 0.150 & 0.156 & 0.00 \\
Central pH level & 6.389 & 6.385 & 6.364 & 6.00 \\
\hline \hline
\end{tabular}




\section{CAPTIONS}

Figure 1 A one micron section of a tumour spheroid showing the inner necrotic region and the outer living region (rat osteogenic sarcoma, reproduced with permission from Yu et al. (2007).)

Figure 2 The 2D lattice structure assumed in the model. Each site is assumed to be occupied by $\gg 1$ individual cells as described in the text. The lattice size is calculated directly from the packing density of tumour cells and the key control parameter - the number of cells per lattice site.

Figure 3 Metabolism decision flow chart. $n_{i}, o_{i}$ and $p H_{i}$ stand for the environmental [CHO], $\left[\mathrm{O}_{2}\right]$ and $\mathrm{pH}$ level at lattice site $i$, respectively. Description of all parameters given in Table 1. 
Figure 4 Division decision flow chart. Successful division requires that a site accrue proliferation time in accordance with its mitotic cycle time and that an adjacent free site exists.

Figure 5 Example growth sequence for typical model run at the end of days as indicated. Medium [CHO], $\left[\mathrm{O}_{2}\right.$ ] and $\mathrm{pH}$ level were set to $16.5 \mathrm{mM}, 0.28 \mathrm{mM}$ and 7.4 , respectively. Colours correspond to site states: aerobic proliferation (black); anaerobic proliferation (red); aerobic quiescence (orange); and anaerobic quiescence (yellow). Unfilled sites are coloured white.

Figure $6 \mathrm{~A}$ linear relationship (after the early transient phase) between $C(t) / N$ and $t^{2}$ as predicted by the peripheral mitotic $C A$ model. Data presented are the mean for two model runs at cell-per-site values $N \in\{10,100,400\}\left(n_{e x}=16.5 \mathrm{mM}, o_{e x}=0.28 \mathrm{mM}\right)$. Broken line added as a guide to the eye. 
Figure 7 Mean bulk growth characteristics over five independent trials. (a) Tumour diameter versus time for $n_{e x}=16.5 \mathrm{mM}, o_{e x}=0.28 \mathrm{mM}$ and $p H_{e x}=7.4$, error bars indicate upper and lower standard deviations for representative points at early and late model time. Line of fit represents a simple linear regression (slope $104.3 \mu \mathrm{m} /$ day, $\left.R^{2}=0.993\right)$. (b) Cell count versus time at $p H_{e x}=7.4, o_{e x}=0.28 \mathrm{mM}$ and $n_{e x}$ as indicated. Lines correspond to non-linear Gompertz equation fits to all data, see text and Table 2 for details. Compare Fig. 1 (p.519) in Freyer and Sutherland (1985)

Figure 8 Typical tumour forms at onset of necrosis under $p H_{e x}=7.4, o_{e x}=0.28 \mathrm{mM}$ and $n_{e x}$ as indicated. Colours indicate state of each site as in Fig. 5.

Figure 9 Percent of cells in each state versus tumour diameter $\left(p H_{e x}=7.4, n_{e x}=\right.$ $\left.16.5 \mathrm{mM}, o_{e x}=0.28 \mathrm{mM}\right)$, mean of five trials: $\mathbf{p}$ - aerobic proliferation; $\mathbf{p}_{\mathrm{an}}$ - anaerobic proliferation; q - aerobic quiescence; and qan - anaerobic quiescence. Compare Fig. 6 (p.522) in Freyer and Sutherland (1985). 
Figure 10 Normalised central $\mathrm{CHO}, \mathrm{O}_{2}$ concentrations and $\mathrm{pH}$ versus tumour diameter $\left(p H_{e x}=7.4, n_{e x}=16.5 \mathrm{mM}, o_{e x}=0.28 \mathrm{mM}\right)$. Average data from five trials. Vertical line indicates the mean onset of necrosis. Compare Fig. 8 (p.519) in Walenta et al. (2000). Values of 1 indicate medium concentrations, whilst values of 0 indicate final concentrations, approximately $1.101 \mathrm{mM}, 0.158 \mathrm{mM}$, and 6.37 for central [CHO], [O $\left.\mathrm{O}_{2}\right]$ and $\mathrm{pH}$ respectively.

Figure 11 Mean $\mathrm{CHO}, \mathrm{O}_{2}$ consumption and $\mathrm{H}^{+}$production per viable cell volume as a function of tumour diameter $\left(p H_{e x}=7.4, n_{e x}=16.5 \mathrm{mM}, o_{e x}=0.28 \mathrm{mM}\right)$. Compare $\mathrm{CHO}$ and $\mathrm{H}^{+}$data with Fig. 6(A,B) (p. 517) in Walenta et al. (2000) and $\mathrm{CHO}$ and $\mathrm{O}_{2}$ data with that of Fig. 3(A,B) (p. 520) in Freyer and Sutherland (1985).

Figure 12 Thickness of viable cell rim as a function of tumour diameter under $p H_{e x}$ $=7.4, o_{e x}=0.28 \mathrm{mM}$ and $n_{e x}=2.8 \mathrm{mM}$ (squares), $5.5 \mathrm{mM}$ (diamonds) and $16.5 \mathrm{mM}$ (circles). Lines are simple linear regression lines of best fit. Compare with data reported in Table 3 Freyer and Sutherland (1985) (p.520). Note: Data presented for $n_{e x}=16.5$ $\mathrm{mM}$ in this and subsequent figures taken over 25 days. 
Figure 13 Thickness of viable cell rim versus medium glucose concentration. Each open circle represents mean of data presented in Fig. 12 with standard deviation represented by error bars. Data for the analagous in vitro study as reported in (top of) Fig. 4, Freyer and Sutherland (1986) is shown by filled circles.

Figure 14 Necrotic volume versus tumour diameter, conditions and labels as in Fig. 12. Filled diamonds represent equivalent in vitro data for the analagous study reported in Table 3 (p.520) of Freyer and Sutherland (1985). 
8 FIGURES

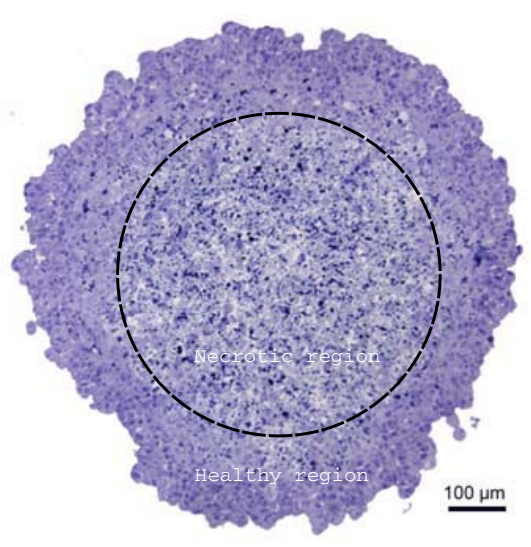

Figure 1

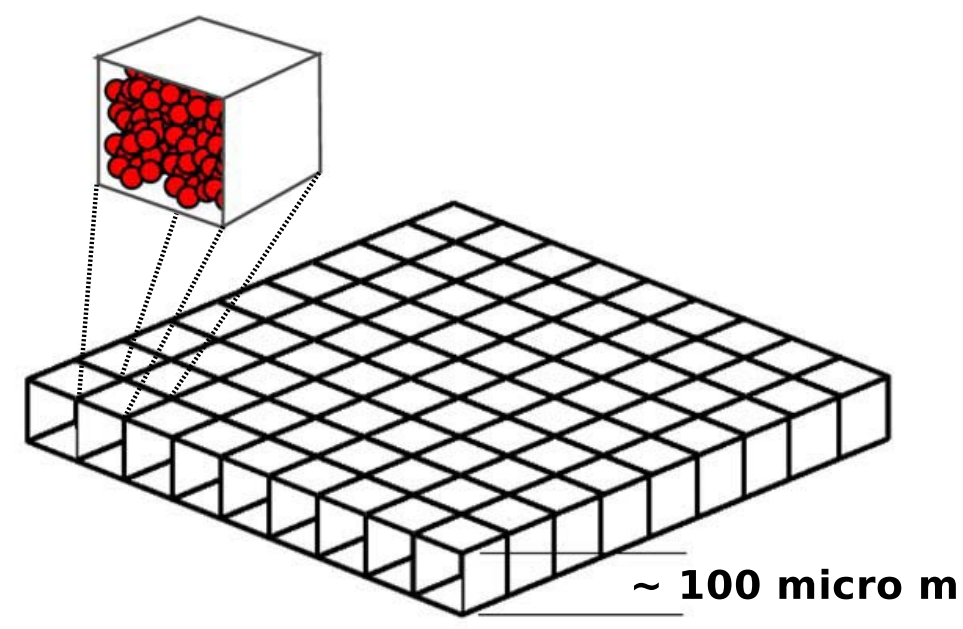

Figure 2 


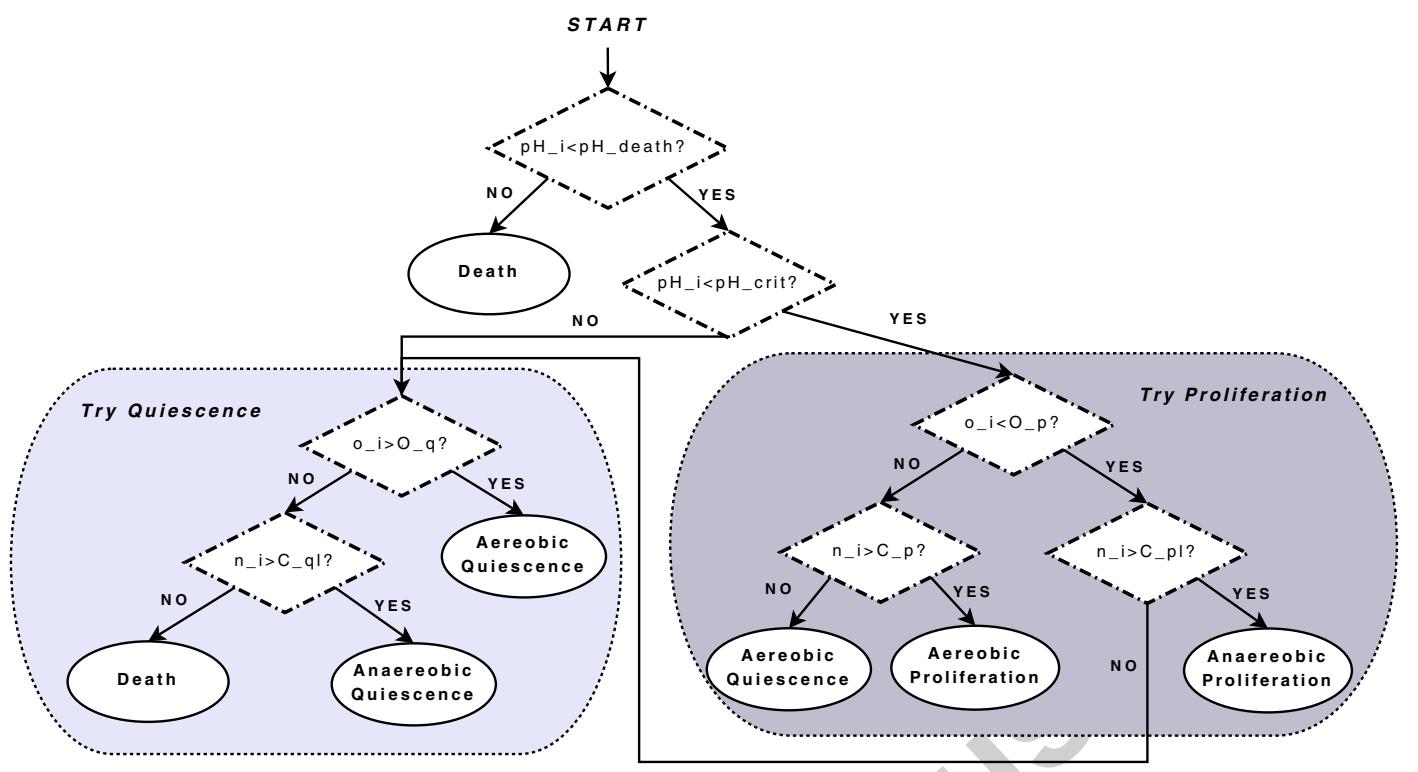

Figure 3

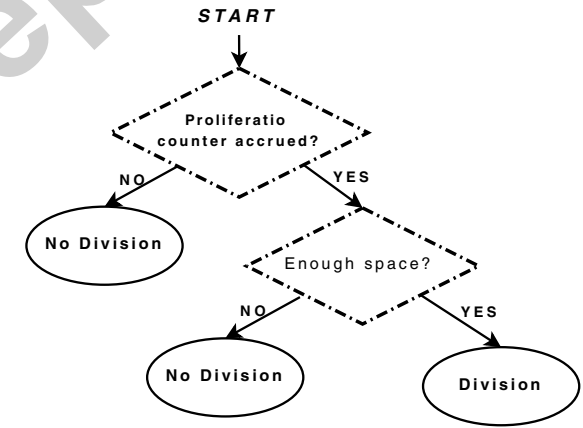

Figure 4 

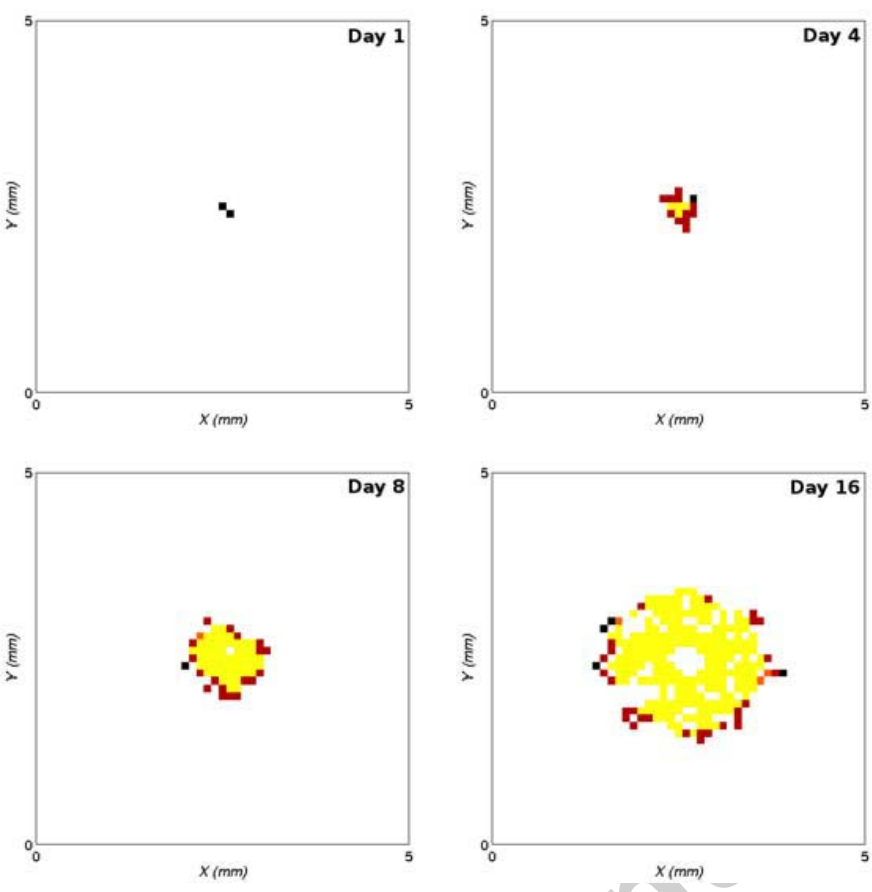

Figure 5

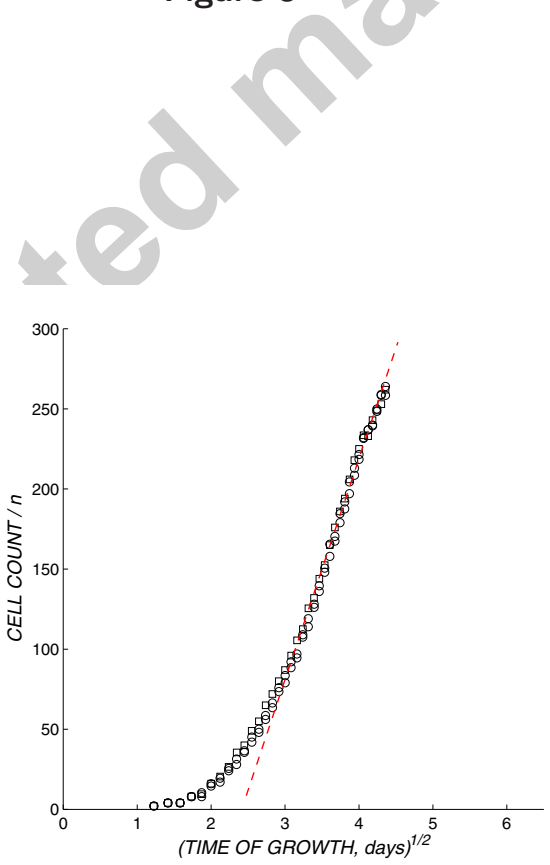

Figure 6 

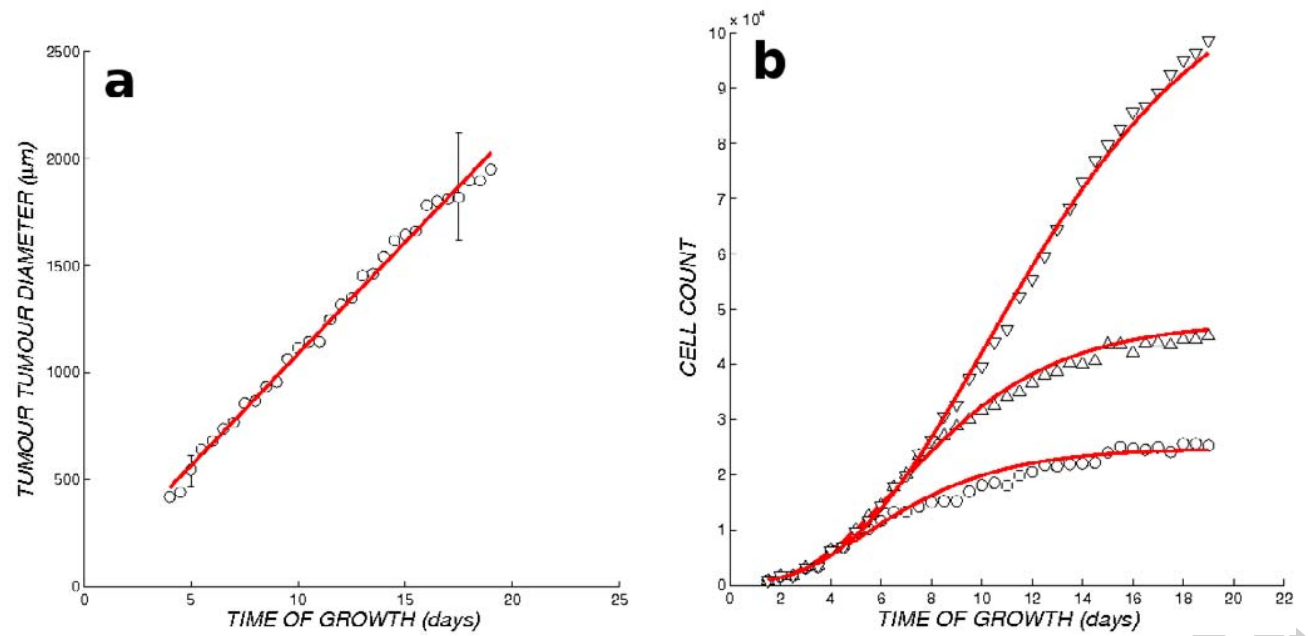

Figure 7
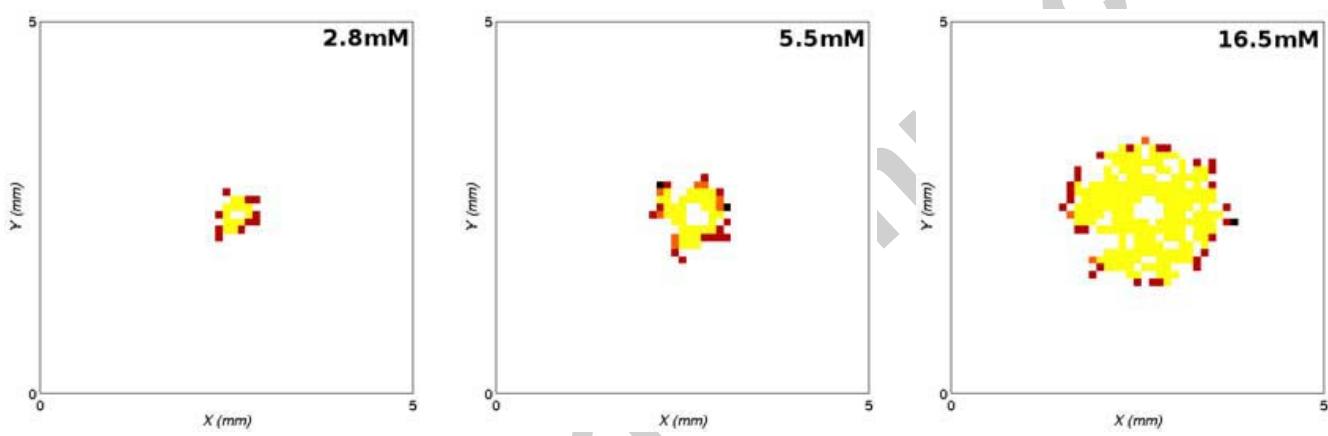

Figure 8

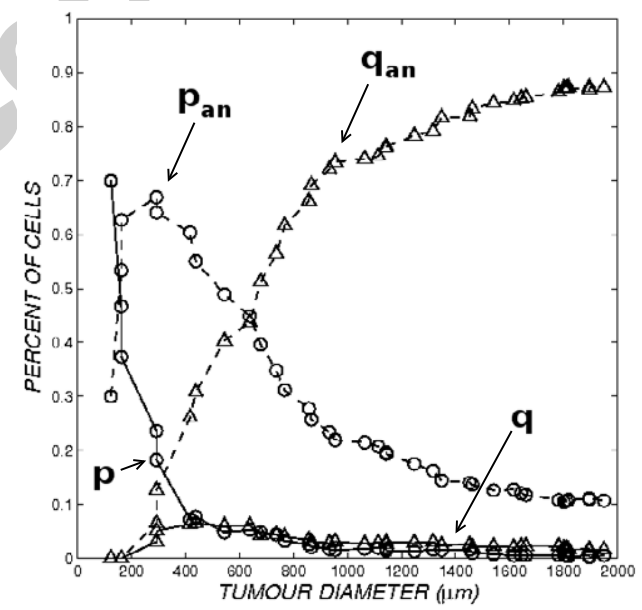

Figure 9 


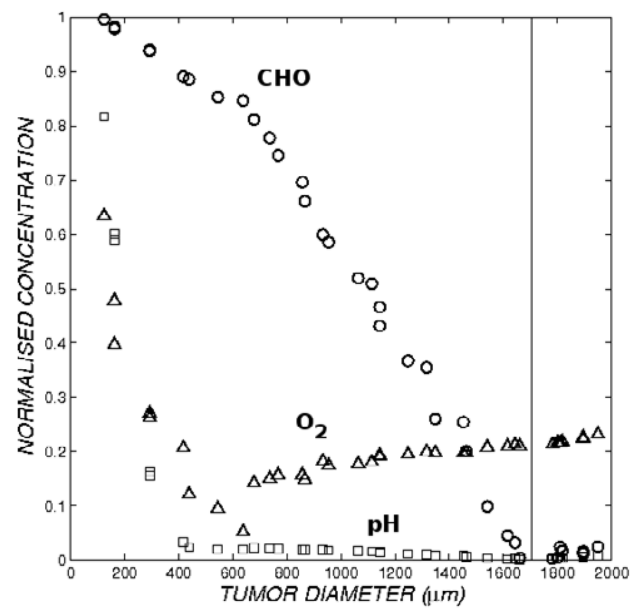

Figure 10

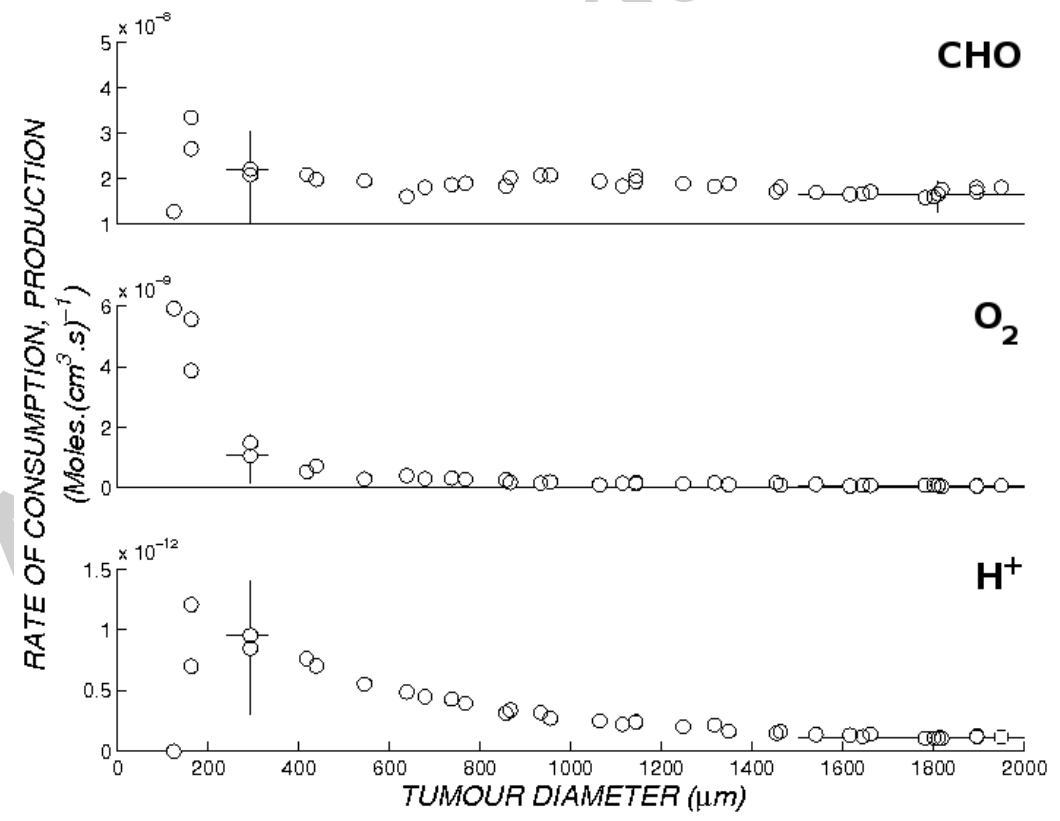

Figure 11 


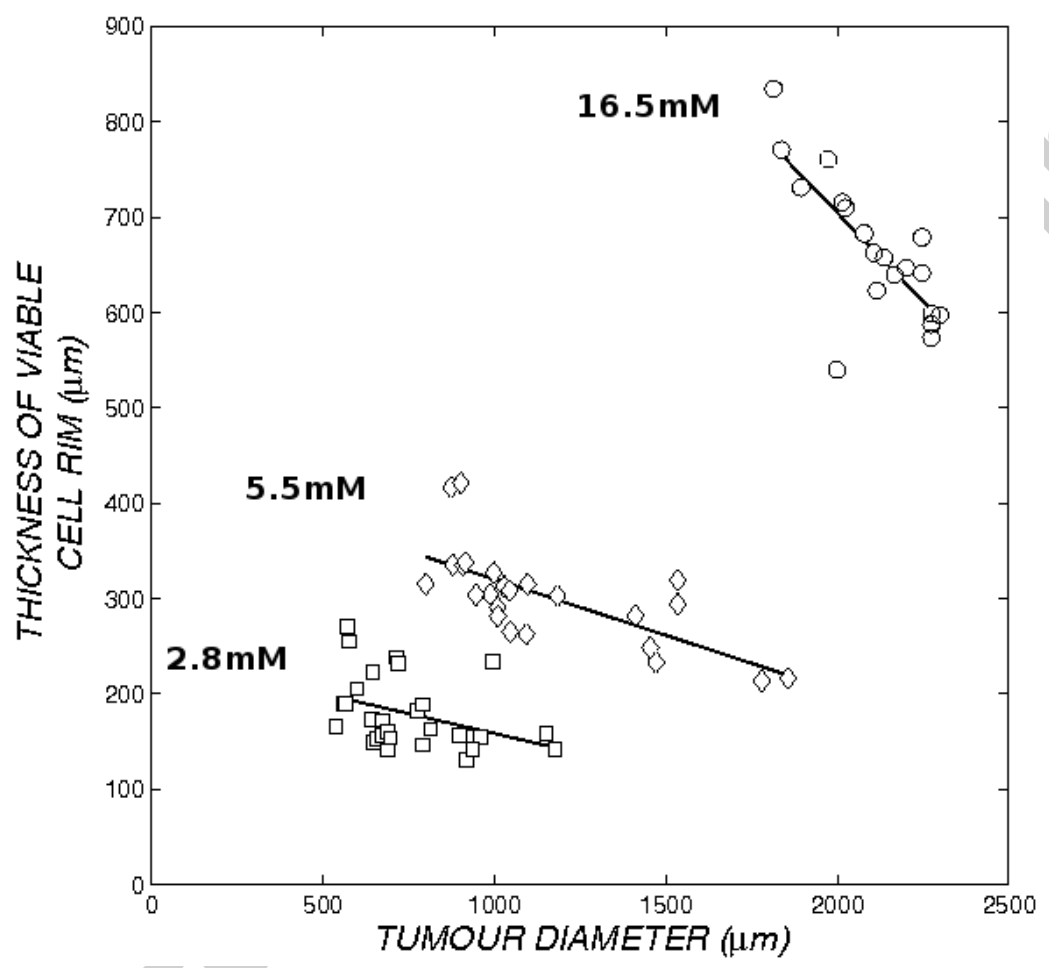

Figure 12 


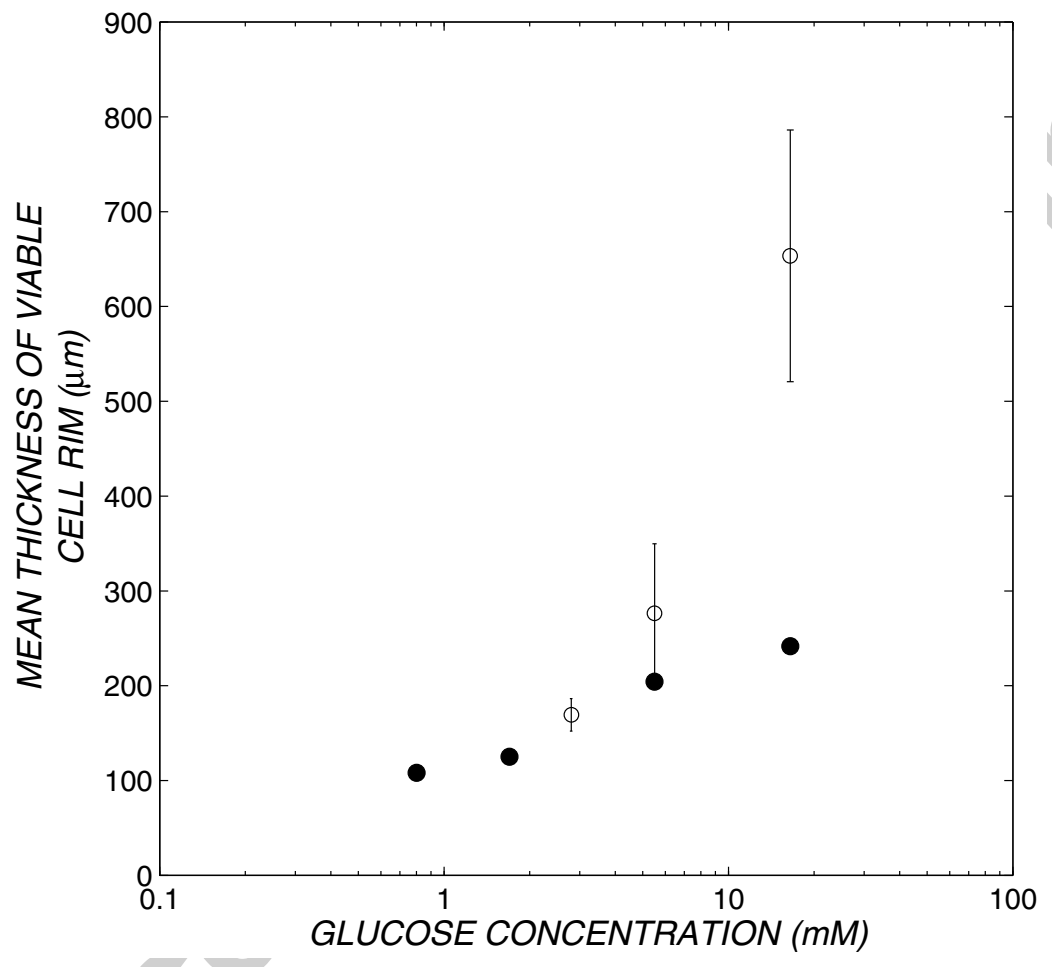

Figure 13 


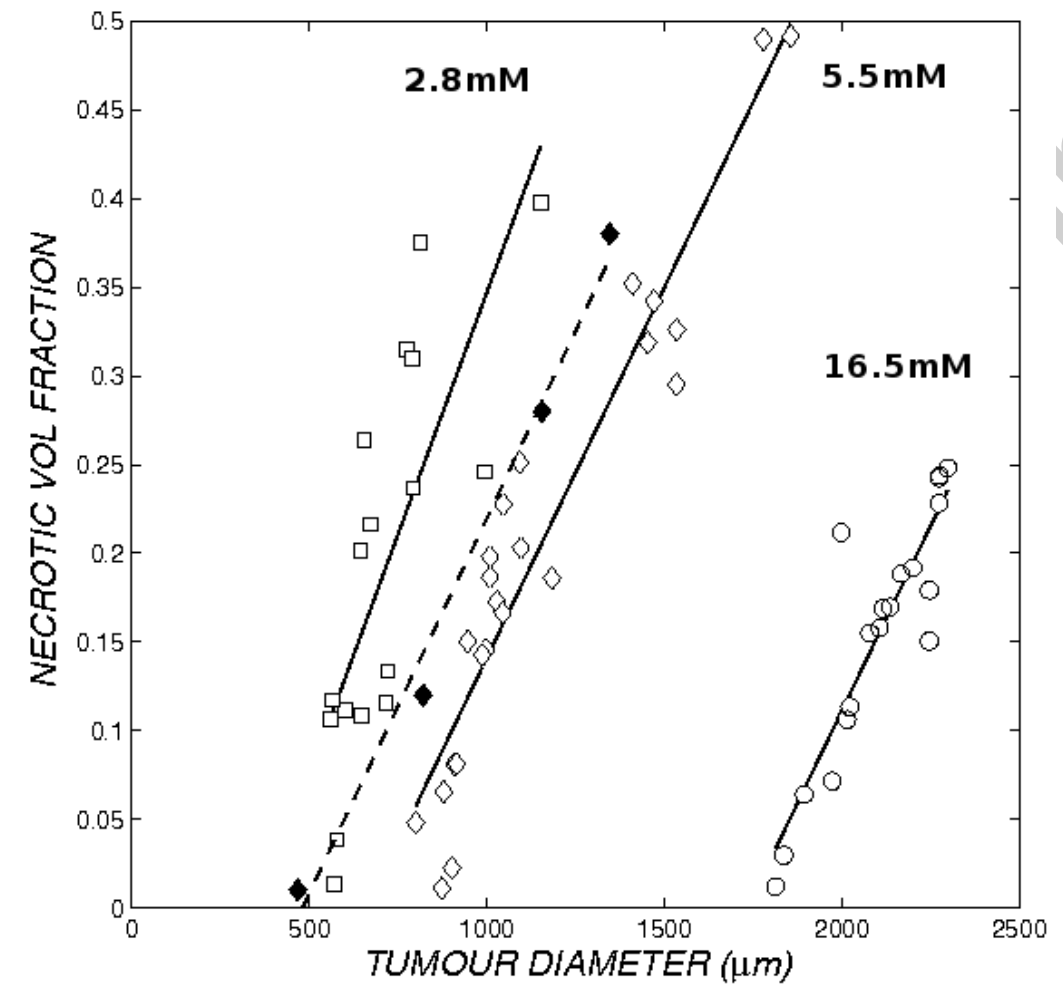

Figure 14 